\title{
Review Article \\ Traditional Chinese Medicine Syndromes for Essential Hypertension: A Literature Analysis of 13,272 Patients
}

\author{
Jie Wang, Xingjiang Xiong, and Wei Liu \\ Department of Cardiology, Guang'anmen Hospital, China Academy of Chinese Medical Sciences, Beijing 100053, China \\ Correspondence should be addressed to Wei Liu; lvzyxxg@163.com
}

Received 29 October 2013; Accepted 19 December 2013; Published 10 February 2014

Academic Editor: Bo Feng

Copyright ( 2014 Jie Wang et al. This is an open access article distributed under the Creative Commons Attribution License, which permits unrestricted use, distribution, and reproduction in any medium, provided the original work is properly cited.

\begin{abstract}
Background. To simplify traditional Chinese medicine syndrome differentiation and allow researchers to master syndrome differentiation for hypertension, this paper retrospectively studied the literature and analyzed syndrome elements corresponding to hypertension syndromes. Methods. Six databases including PubMed, EMBASE, Chinese Bio-Medical Literature Database, Chinese National Knowledge Infrastructure, Chinese Scientific Journal Database, and Wan-fang Data were searched from 1/January/2003 to 30/October/2013. We included all clinical literature testing hypertension syndromes and retrospectively studied the hypertension literature published from 2003 to 2013. Descriptive statistics calculated frequencies and percentages. Results. 13,272 patients with essential hypertension were included. Clinical features of hypertension could be attributed to 11 kinds of syndrome factors. Among them, seven syndrome factors were excess, while four syndrome factors were deficient. Syndrome targets were mainly in the liver and related to the kidney and spleen. There were 33 combination syndromes. Frequency of single-factor syndromes was $31.77 \%$ and frequency of two-factor syndromes was $62.26 \%$. Conclusions. Excess syndrome factors of hypertension patients include yang hyperactivity, blood stasis, phlegm turbidity, internal dampness, and internal fire. Deficient syndrome factors of hypertension patients are yin deficiency and yang deficiency. Yin deficiency with yang hyperactivity, phlegm-dampness retention, and deficiency of both yin and yang were the three most common syndromes in clinical combination.
\end{abstract}

\section{Introduction}

Hypertension is an important public health issue worldwide because of its high prevalence and concomitant increase in disease risk [1-3]. It has been estimated that $29 \%$ of the world's adult population, or approximately 1.56 billion people, will have hypertension by $2025[4,5]$. Complementary and alternative medicine (CAM) is becoming increasingly popular [613] and numerous interventions are regularly recommended to lower elevated blood pressure (BP) [14-17]. Traditional Chinese medicine (TCM), including herbal medicine and acupuncture, is an important component of CAM therapies [18-21]. Hypertension could be improved by insights from TCM and considerable progress has been made in lowering BP by TCM [22-26].

Syndrome differentiation is a diagnostic and treatment method used in TCM $[27,28]$. It plays an important role in the therapeutic process and affects the therapeutic result of certain diseases [29-31]. The syndrome is not only the basic unit of TCM theory and syndrome differentiation, but also the bridge to associating disease and formula [3235]. TCM syndrome, which is different from a disease or symptoms, is the abstraction of a major pathogenesis. Syndromes are identified from a comprehensive analysis of all symptoms and signs (including tongue appearance and pulse feeling) from the four main diagnostic TCM methods: observation, listening, questioning, and pulse analyses [3640]. However, syndromes are the product of speculation in TCM. Therefore, they depend on medical experience, academic origins, and other factors. Therefore, the concept of syndromes is vague and broad, which makes clinical application difficult. Syndrome elements, which are the minimum units of syndromes, contribute to simplifying syndrome differentiation and understanding TCM syndromes. Each element has specific symptoms.

To simplify TCM syndrome differentiation and enable researchers not familiar with Chinese medicine to master the laws of hypertension syndrome differentiation, this paper 
retrospectively studied the literature for 13,272 patients with hypertension, published from 2003 to 2013. This study is beneficial to deepening of the understanding of hypertension and providing a basis and reference for clinical treatment using TCM syndrome differentiation.

\section{Materials and Methods}

2.1. Database and Search Strategies. Six databases including PubMed, EMBASE, Chinese Bio-Medical Literature Database (CBM), Chinese National Knowledge Infrastructure (CNKI), Chinese Scientific Journal Database (VIP), and Wan-fang Data were searched from 1/January/2003 to $30 /$ October/2013. Databases in Chinese were searched to retrieve the maximum possible number of trials of syndrome differentiation for essential hypertension (EH) because syndrome differentiation is mainly used in China. Ongoing registered clinical trials were searched at the International Clinical Trial Registry by the U.S. National Institutes of Health (http://clinicaltrials.gov/). The following search terms were used individually or combined: "hypertension," "blood pressure," "essential hypertension," "syndrome differentiation," "vertigo," "headache," "parting," and "traditional Chinese medicine therapy." The bibliographies of included studies were searched for additional references.

2.2. Inclusion and Exclusion Criteria. Systolic blood pressure $(\mathrm{SBP}) \geq 140 \mathrm{mmHg}(1 \mathrm{mmHg}=0.133 \mathrm{kPa})$ and diastolic blood pressure (DBP) $\geq 90 \mathrm{mmHg}$ from the literature were based on 1999 WHO-ISH Guidelines for the Management of Hypertension (1999 WHO-ISH GMH), 1998 WHO-ISH Guidelines for the Management of Hypertension (1998 WHO-ISH GMH), 2000 WHO-ISH Guidelines for the Management of Hypertension (2000 WHO-ISH GMH), Chinese Guidelines for the Management of Hypertension2005 (CGMH-2005), China Guidelines on Prevention and Management of High Blood Pressure-2006 (CGPMHBP2006), and Seventh Report of the Joint National Committee on Prevention, Detection, Evaluation, and Treatment of High Blood Pressure (JNC 7). Syndrome differentiation of TCM diagnosis used the Standard of TCM Diagnosis and Curative Effect of Disease-Syndrome, published by the State Administration of Traditional Chinese Medicine in 1994. Standards of dialectical classification used Clinical Research Guiding Principles of New Medicine of Chinese Herbs revised by the State Food and Drug Administration in 2002. Exclusion criteria were secondary hypertension, gestational hypertension, repeated literature, reviews, and literature with no clear classification.

2.3. Classification Criteria of Syndrome Elements. According to the classification criteria of syndrome elements proposed by Wang, statistical analysis was conducted for syndromes included in the cases. The classification criteria of syndrome elements were (1) six-excess external contraction: wind, cold, dampness, dryness, and fire; (2) five endogenous qi: internal wind, internal cold, internal dampness, internal dryness, and internal fire; (3) factors related to gas: qi deficiency, qi stagnation, qi block, qi counterflow, qi fall, and qi collapse; (4) factors related to blood: blood deficiency, blood stasis, blood collapse, blood dryness, and bleeding; (5) factors related to yin and yang: yin deficiency, yang deficiency, yin exuberance, and yang hyperactivity; (6) others: poison, excessive fluid, and phlegm turbidity.

2.4. Statistical Methods. Two authors conducted the literature search, study selection, and data extraction independently. Disagreements were resolved by discussion and consensus was met through a third party. SPSS 11.5 statistical software was used for data analyses (Chicago, IL, USA). Descriptive statistics procedures calculated frequency and percentage.

\section{Results}

3.1. Description of Included Literature. After a primary search of the databases, 503 articles were screened. After reading the titles and abstracts, 398 articles were excluded the reasons included; retrospective study that did not match the included criteria of this review $(n=42)$ and duplicated titles $(n=356)$. The full texts of 83 articles [41-123] were retrieved, and 22 articles were excluded for the following reasons: participants not meeting the inclusion criteria $(n=11)$, duplicated data $(n=5)$, patients having other diseases $(n=5)$, and no data for extraction $(n=1)$. In the end, 83 articles [41-123] were included, and all trials were conducted in China (Figure 1). The characteristics of included trials are listed in Table 1.

Overall, 13,272 patients with essential hypertension were included, with an average of 160 per trial, ranging from 23 to 703 . Among them, 7075 were men, accounting for $53.3 \%$, while 6197 were women, accounting for $46.7 \%$. There was a wide range in patient age (18-92 years). Sources of cases included 24 provinces and the number of papers in each region is shown in Table 2.

3.2. Extraction of Syndrome Elements of EH. According to the definition of syndrome elements and classification criteria, syndrome elements were obtained and classified from the literature as follows: blood stasis (qi stagnation and blood stasis, qi deficiency with blood stasis, kidney deficiency and blood stasis, stasis blocking channels, phlegm and blood stasis resistance winding); qi stagnation (liver qi stagnation, qi stagnation and blood stasis); phlegm (phlegm turbidity resistance, phlegm-dampness retention); internal fire (intense liver fire, internal harassment of phlegm-heat); internal dampness (spleen deficiency with dampness encumbrance, phlegm-damp retention); internal wind (internal stirring of liver wind, wind-yang interference); qi deficiency (dual deficiency of qi and yin, dual deficiency of qi and blood, and qi deficiency with blood stasis); yang hyperactivity (ascendant hyperactivity of liver yang, yin deficiency with yang hyperactivity); yin deficiency (yin deficiency with yang hyperactivity, liver-kidney yin deficiency, dual deficiency of qi and yin, and deficiency of both yin and yang); yang deficiency (kidney yang deficiency, deficiency of both yin and yang); blood deficiency (dual deficiency of qi and blood). 


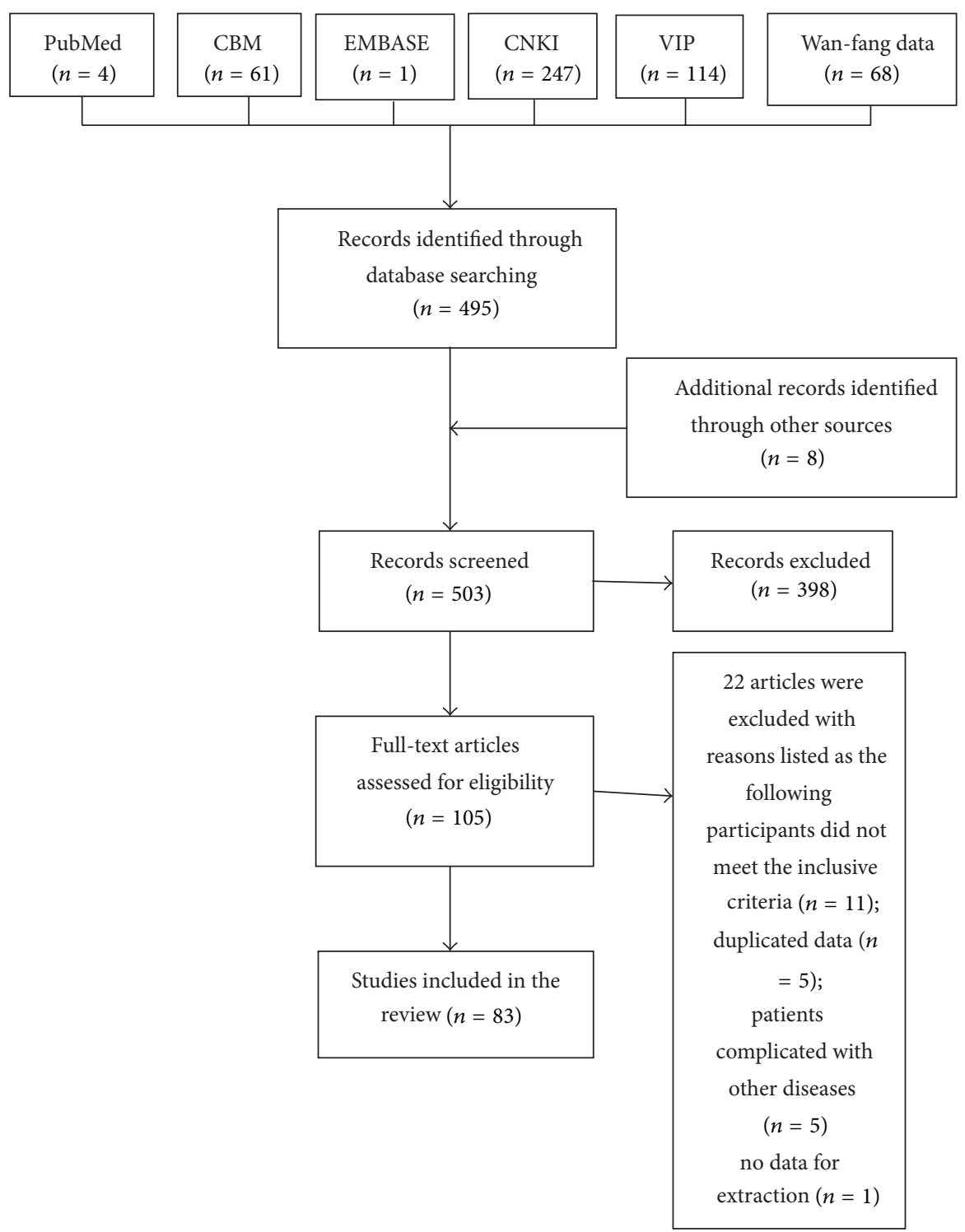

FIGURE 1: Screening process of articles.

As a result, 13,272 cases of hypertension syndrome were classified as 11 syndrome element types, which cover all cases.

3.3. Analysis of Syndrome Elements of EH. Syndrome elements of 13,272 patients with hypertension were divided into excessive syndrome elements and deficient syndrome elements (Table 3, Figure 2). The proportions of excessive syndrome elements are yang hyperactivity (19.08\%), phlegm turbidity $(13.68 \%)$, internal fire $(13.21 \%)$, internal dampness $(11.04 \%)$, blood stasis $(4.86 \%)$, internal wind $(1.21 \%)$, and qi stagnation $(0.78 \%)$. The proportion of deficient syndrome elements are yin deficiency (26.27\%), yang deficiency $(7.89 \%)$, qi deficiency $(1.80 \%)$, and blood deficiency $(0.18 \%)$. Excessive syndrome elements greater than $10 \%$ included yang hyperactivity, phlegm turbidity, internal fire, and internal dampness. Deficient syndrome elements greater than $10 \%$ included yin deficiency. Yang hyperactivity and yin deficiency were the most common syndrome elements of hypertension.

3.4. Targets of Syndrome Elements of EH. The targets of syndrome elements are the disease locations of individual syndrome elements. Disease location of syndrome elements was confirmed according to the five zang-organs and six fuorgans, chi heng fu, and meridians.

As a result, 9091 cases $(68.50 \%)$ had clear targets of syndrome elements related to liver, kidney, and spleen (Table 4). There were 7789 cases of liver syndromes (85.68\%). Among them, there were 2793 cases of internal fire of liver $(35.86 \%)$, 4033 cases of ascendant hyperactivity of liver yang (51.78\%), 543 cases of liver yin deficiency (6.97\%), 164 cases of liver qi stagnation $(2.11 \%)$, and 256 cases of internal stirring of liver wind (3.29\%). There were 903 cases of kidney syndromes 
TABLE 1: Characteristics of included studies.

\begin{tabular}{|c|c|c|c|c|c|}
\hline Study ID & $\begin{array}{l}\text { Sample } \\
(\mathrm{M} / \mathrm{F})\end{array}$ & Age (years) & Diagnosis standard & $\begin{array}{l}\text { TCM syndrome differentiation } \\
\text { (number of patients) }\end{array}$ & $\begin{array}{c}\text { Region of } \\
\text { China }\end{array}$ \\
\hline $\begin{array}{l}\text { Xu and Chen } \\
2012[13]\end{array}$ & $\begin{array}{c}122 \\
(58 / 64)\end{array}$ & $60-79$ & $\begin{array}{l}\text { Chinese Guidelines for the } \\
\text { Management of } \\
\text { Hypertension-2005 } \\
\text { (CGMH-2005) }\end{array}$ & $\begin{array}{l}\text { Phlegm and blood stasis resistance winding } \\
\text { (39), yin deficiency with yang hyperactivity } \\
\text { (44), and idney deficiency (39) }\end{array}$ & Beijing \\
\hline $\begin{array}{l}\text { Ferreira and } \\
\text { Lopes } 2011[14]\end{array}$ & $\begin{array}{c}448 \\
(243 / 205)\end{array}$ & $\begin{array}{c}\text { M: } 62.1 \pm 10.9 \\
\text { F: } 59.3 \pm 8.7\end{array}$ & $\begin{array}{l}\text { Chinese Guidelines for the } \\
\text { Management of } \\
\text { Hypertension-2005 } \\
\text { (CGMH-2005) }\end{array}$ & $\begin{array}{l}\text { Intense liver fire (284), yin deficiency with } \\
\text { yang hyperactivity (43), phlegm-damp } \\
\text { retention ( } 74) \text {, and deficiency of both yin } \\
\text { and yang ( } 47)\end{array}$ & Jiangsu \\
\hline $\begin{array}{l}\text { Wang et al. } \\
2013[15]\end{array}$ & $\begin{array}{c}99 \\
(50 / 49)\end{array}$ & $73 \pm 6.1$ & 1999 WHO-ISH GMH & Qi stagnation and blood stasis (99) & Guangdong \\
\hline $\begin{array}{l}\text { Lee et al. } \\
2004[16]\end{array}$ & $\begin{array}{c}87 \\
(48 / 39)\end{array}$ & $\begin{array}{r}\text { M: } 62.7 \pm 8.3 \\
\text { F: } 58.9 \pm 7.5\end{array}$ & $\begin{array}{l}\text { Chinese Guidelines for the } \\
\text { Management of } \\
\text { Hypertension-2005 } \\
\text { (CGMH-2005) }\end{array}$ & $\begin{array}{l}\text { Ascendant hyperactivity of liver yang ( } 32) \text {, } \\
\text { phlegm-damp retention (27), and qi } \\
\text { deficiency with blood stasis (28) }\end{array}$ & Guangdong \\
\hline $\begin{array}{l}\text { Wang et al. } \\
2013[17]\end{array}$ & $\begin{array}{c}140 \\
(83 / 57)\end{array}$ & $56.5 \pm 9.8$ & $\begin{array}{l}\text { Chinese Guidelines for the } \\
\text { Management of } \\
\text { Hypertension-2010 } \\
\text { (CGMH-2010) }\end{array}$ & $\begin{array}{l}\text { Intense liver fire (28), yin deficiency with } \\
\text { yang hyperactivity (39), phlegm-damp } \\
\text { retention (45), and deficiency of both yin } \\
\text { and yang (28) }\end{array}$ & Heibei \\
\hline $\begin{array}{l}\text { Wang and Xiong } \\
2012[18]\end{array}$ & $\begin{array}{c}76 \\
(38 / 38)\end{array}$ & Not reported & $\begin{array}{l}\text { Chinese Guidelines for the } \\
\text { Management of } \\
\text { Hypertension-2010 } \\
\text { (CGMH-2010) }\end{array}$ & Kidney deficiency and blood stasis (76) & Fujian \\
\hline $\begin{array}{l}\text { Xiong et al. } \\
2013[19]\end{array}$ & $\begin{array}{c}395 \\
(228 / 167)\end{array}$ & $53 \pm 17$ & $\begin{array}{l}\text { Hypertension diagnostic } \\
\text { criteria (unclear) }\end{array}$ & $\begin{array}{l}\text { Intense liver fire (54), yin deficiency with } \\
\text { yang hyperactivity (177), phlegm-damp } \\
\text { retention (62), and deficiency of both yin } \\
\text { and yang (102) }\end{array}$ & Liaoning \\
\hline $\begin{array}{l}\text { Wang et al. } \\
2013[20]\end{array}$ & $\begin{array}{c}120 \\
(60 / 60)\end{array}$ & $29-62$ & $\begin{array}{l}\text { Hypertension diagnostic } \\
\text { criteria (unclear) }\end{array}$ & $\begin{array}{l}\text { Ascendant hyperactivity of liver yang ( } 30) \text {, } \\
\text { yin deficiency with yang hyperactivity (30), } \\
\text { phlegm-damp retention ( } 30) \text {, and deficiency } \\
\text { of both yin and yang (30) }\end{array}$ & Hainan \\
\hline $\begin{array}{l}\text { Wang et al. } 2013 \\
\text { [21] }\end{array}$ & $\begin{array}{c}184 \\
(83 / 101)\end{array}$ & $18-80$ & $\begin{array}{l}\text { Chinese Guidelines for the } \\
\text { Management of } \\
\text { Hypertension-2005 } \\
\text { (CGMH-2005) }\end{array}$ & $\begin{array}{l}\text { Intense liver fire (21), yin deficiency with } \\
\text { yang hyperactivity ( } 75) \text {, phlegm-damp } \\
\text { retention ( } 81) \text {, and deficiency of both yin } \\
\text { and yang ( } 7 \text { ) }\end{array}$ & Jiangsu \\
\hline Chen 1993 [22] & $\begin{array}{c}60 \\
(30 / 30)\end{array}$ & $\begin{array}{l}\mathrm{T}: 48 \pm 8.1 \\
\mathrm{C}: 47 \pm 6.7\end{array}$ & 1999 WHO-ISH GMH & Dual deficiency of qi and yin (60) & Guangdong \\
\hline $\begin{array}{l}\text { Wang et al. } 2012 \\
\text { [23] }\end{array}$ & $\begin{array}{c}53 \\
(16 / 37)\end{array}$ & $40-80$ & 1999 WHO-ISH GMH & $\begin{array}{l}\text { Insufficiency of spleen with overabundance } \\
\text { of dampness (19), dual deficiency of qi and } \\
\text { blood (20), and liver-kidney yin deficiency } \\
\text { (14) }\end{array}$ & Neimenggu \\
\hline $\begin{array}{l}\text { Wang et al. } \\
2013[24]\end{array}$ & $112(83 / 29)$ & $53.5 \pm 11.04$ & 1999 WHO-ISH GMH & $\begin{array}{l}\text { Intense liver fire (19), yin deficiency with } \\
\text { yang hyperactivity (23), phlegm-damp } \\
\text { retention (16), and deficiency of both yin } \\
\text { and yang (22) }\end{array}$ & Jiangxi \\
\hline $\begin{array}{l}\text { Wang and Xiong } \\
2012[25]\end{array}$ & $\begin{array}{l}61 \\
(\mathrm{M} / \mathrm{F} \text { not } \\
\text { reported })\end{array}$ & $\begin{array}{l}\text { T: } 57.1 \pm 6.16 \\
\text { C: } 55.67 \pm 6.28\end{array}$ & $\begin{array}{l}\text { Chinese Guidelines for the } \\
\text { Management of } \\
\text { Hypertension-2004 } \\
\text { (CGMH-2004) }\end{array}$ & Blood stasis (61) & Guangdong \\
\hline $\begin{array}{l}\text { Chen et al. } 2011 \\
{[26]}\end{array}$ & $\begin{array}{c}259 \\
(108 / 151)\end{array}$ & $65.58 \pm 12.17$ & $\begin{array}{l}\text { Chinese Guidelines for the } \\
\text { Management of } \\
\text { Hypertension-2005 } \\
\text { (CGMH-2005) }\end{array}$ & $\begin{array}{l}\text { Intense liver fire (35), yin deficiency with } \\
\text { yang hyperactivity (89), phlegm-damp } \\
\text { retention (88), and deficiency of both yin } \\
\text { and yang (47) }\end{array}$ & Beijing \\
\hline $\begin{array}{l}\mathrm{Xu} \text { and Chen } \\
2011 \text { [27] }\end{array}$ & $\begin{array}{c}81 \\
(53 / 28)\end{array}$ & $52.79 \pm 12.83$ & $\begin{array}{l}\text { Chinese Guidelines for the } \\
\text { Management of } \\
\text { Hypertension-2005 } \\
(\text { CGMH-2005) }\end{array}$ & $\begin{array}{l}\text { Intense liver fire (42), yin deficiency with } \\
\text { yang hyperactivity (18), phlegm-damp } \\
\text { retention (14), and deficiency of both yin } \\
\text { and yang ( } 7 \text { ) }\end{array}$ & Zhejiang \\
\hline
\end{tabular}


TABle 1: Continued.

\begin{tabular}{|c|c|c|c|c|c|}
\hline Study ID & $\begin{array}{l}\text { Sample } \\
(\mathrm{M} / \mathrm{F})\end{array}$ & Age (years) & Diagnosis standard & $\begin{array}{l}\text { TCM syndrome differentiation } \\
\text { (number of patients) }\end{array}$ & $\begin{array}{l}\text { Region of } \\
\text { China }\end{array}$ \\
\hline $\begin{array}{l}\text { Chen et al. } \\
2012[28]\end{array}$ & $\begin{array}{c}183 \\
(85 / 98)\end{array}$ & $66.81 \pm 8.81$ & $\begin{array}{l}\text { Chinese Guidelines for the } \\
\text { Management of } \\
\text { Hypertension-2004 } \\
\text { (CGMH-2004) }\end{array}$ & $\begin{array}{l}\text { Intense liver fire (28), yin deficiency with } \\
\text { yang hyperactivity (53), phlegm-damp } \\
\text { retention (57), and deficiency of both yin } \\
\text { and yang (45) }\end{array}$ & Jiangsu \\
\hline $\begin{array}{l}\text { Liu et al. } \\
2011[29]\end{array}$ & $\begin{array}{c}89 \\
(45 / 44)\end{array}$ & $\begin{array}{l}\text { M: } 59.5 \pm 10.9 \\
\text { F: } 59.3 \pm 11.0\end{array}$ & $\begin{array}{l}\text { Chinese Guidelines for the } \\
\text { Management of } \\
\text { Hypertension-2005 } \\
\text { (CGMH-2005) }\end{array}$ & $\begin{array}{l}\text { Intense liver fire (59), yin deficiency with } \\
\text { yang hyperactivity (5), phlegm-damp } \\
\text { retention (13), and deficiency of both yin } \\
\text { and yang (12) }\end{array}$ & Jiangsu \\
\hline $\begin{array}{l}\text { Dobos and Tao } \\
2011[30]\end{array}$ & $\begin{array}{c}342 \\
(213 / 129)\end{array}$ & $\begin{array}{l}\text { M: } 59.43 \pm 16.76 \\
\text { F: } 59.43 \pm 11.82\end{array}$ & 1999 WHO-ISH GMH & $\begin{array}{l}\text { Intense liver fire (51), yin deficiency with } \\
\text { yang hyperactivity (139), phlegm-damp } \\
\text { retention (85), and deficiency of both yin } \\
\text { and yang (67) }\end{array}$ & Guangdong \\
\hline $\begin{array}{l}\text { Xiong et al. } \\
2011[31]\end{array}$ & $\begin{array}{c}562 \\
(297 / 265)\end{array}$ & $\begin{array}{c}\text { M: } 62.1 \pm 10.8 \\
\text { F: } 58.5 \pm 9.1\end{array}$ & $\begin{array}{l}\text { Chinese Guidelines for the } \\
\text { Management of } \\
\text { Hypertension-2005 } \\
\text { (CGMH-2005) }\end{array}$ & $\begin{array}{l}\text { Intense liver fire ( } 352 \text { ), yin deficiency with } \\
\text { yang hyperactivity (58), phlegm-damp } \\
\text { retention (97), and deficiency of both yin } \\
\text { and yang (55) }\end{array}$ & Jiangsu \\
\hline $\begin{array}{l}\text { Wang and } \\
\text { Xiong } 2012[32]\end{array}$ & $\begin{array}{c}398 \\
(199 / 199)\end{array}$ & $59.20 \pm 9.54$ & $\begin{array}{l}\text { Chinese Guidelines for the } \\
\text { Management of } \\
\text { Hypertension-2005 } \\
\text { (CGMH-2005) }\end{array}$ & $\begin{array}{l}\text { Intense liver fire (88), yin deficiency with } \\
\text { yang hyperactivity (196), phlegm-damp } \\
\text { retention (89), and deficiency of both yin } \\
\text { and yang (25) }\end{array}$ & Jiangsu \\
\hline $\begin{array}{l}\text { Wang et al. } 2013 \\
\text { [33] }\end{array}$ & $\begin{array}{c}178 \\
(81 / 97)\end{array}$ & $18-80$ & $\begin{array}{l}\text { Chinese Guidelines for the } \\
\text { Management of } \\
\text { Hypertension-2005 } \\
\text { (CGMH-2005) }\end{array}$ & $\begin{array}{l}\text { Intense liver fire (49), yin deficiency with } \\
\text { yang hyperactivity ( } 43 \text { ), phlegm-damp } \\
\text { retention (57), and deficiency of both yin } \\
\text { and yang (29) }\end{array}$ & Shanghai \\
\hline Tian $2011[34]$ & $\begin{array}{c}200 \\
(109 / 91)\end{array}$ & $30-75$ & 1999 WHO-ISH GMH & $\begin{array}{l}\text { Intense liver fire (37), yin deficiency with } \\
\text { yang hyperactivity (55), phlegm-damp } \\
\text { retention (82), and deficiency of both yin } \\
\text { and yang (26) }\end{array}$ & Tianjin \\
\hline $\begin{array}{l}\text { Wang and } \\
\text { Xiong } 2012 \text { [35] }\end{array}$ & $\begin{array}{l}120 \\
64 / 56\end{array}$ & $\begin{array}{l}\text { T: } 62.77 \pm 9.18 \\
\text { C: } 59.63 \pm 8.77\end{array}$ & 1999 WHO-ISH GMH & $\begin{array}{l}\text { Intense liver fire (37), yin deficiency with } \\
\text { yang hyperactivity (55), phlegm-damp } \\
\text { retention (82), and deficiency of both yin } \\
\text { and yang (26) }\end{array}$ & Hunan \\
\hline $\begin{array}{l}\text { Wang et al. } 2012 \\
{[36]}\end{array}$ & $\begin{array}{c}494 \\
(264 / 230)\end{array}$ & $\begin{array}{c}\text { M: } 61.6 \pm 10.6 \\
\text { F: } 58.3 \pm 8.5\end{array}$ & $\begin{array}{l}\text { Chinese Guidelines for the } \\
\text { Management of } \\
\text { Hypertension-2005 } \\
\text { (CGMH-2005) }\end{array}$ & $\begin{array}{l}\text { Ascendant hyperactivity of liver yang ( } 313) \text {, } \\
\text { yin deficiency with yang hyperactivity ( } 52) \text {, } \\
\text { deficiency of both yin and yang (83), and } \\
\text { liver-kidney yin deficiency (46) }\end{array}$ & Jiangsu \\
\hline $\begin{array}{l}\text { Xu and Chen } \\
2008 \text { [37] }\end{array}$ & $\begin{array}{l}150 \\
\text { (M/F not } \\
\text { reported) }\end{array}$ & Not reported & $\begin{array}{l}\text { Chinese Guidelines for the } \\
\text { Management of } \\
\text { Hypertension-2005 } \\
\text { (CGMH-2005) }\end{array}$ & $\begin{array}{l}\text { Intense liver fire (29), randomized } \\
\text { stagnation of phlegm (53), dual deficiency of } \\
\text { qi and yin (30), and stasis blocking channels } \\
\text { (38) }\end{array}$ & Xinjiang \\
\hline $\begin{array}{l}\text { Cheung } 2011 \\
{[38]}\end{array}$ & $\begin{array}{c}109 \\
(68 / 41)\end{array}$ & $65.6 \pm 10.6$ & 1999 WHO-ISH GMH & $\begin{array}{l}\text { Intense liver fire (19), yin deficiency with } \\
\text { yang hyperactivity (18), phlegm-damp } \\
\text { retention (34), and deficiency of both yin } \\
\text { and yang (38) }\end{array}$ & Fujian \\
\hline $\begin{array}{l}\text { Xiong et al. } 2013 \\
\text { [39] }\end{array}$ & $\begin{array}{c}102 \\
(58 / 44)\end{array}$ & $37-85$ & 1999 WHO-ISH GMH & $\begin{array}{l}\text { Intense liver fire (18), yin deficiency with } \\
\text { yang hyperactivity (31), phlegm-damp } \\
\text { retention (23), and deficiency of both yin } \\
\text { and yang ( } 30)\end{array}$ & Guizhou \\
\hline $\begin{array}{l}\text { Lu et al. } \\
2004 \text { [40] }\end{array}$ & $\begin{array}{c}40 \\
(23 / 17)\end{array}$ & Not reported & 1999 WHO-ISH GMH & Blood stasis (40) & Guangdong \\
\hline $\begin{array}{l}\text { Zhao et al. } 2012 \\
\text { [41] }\end{array}$ & $\begin{array}{c}60 \\
(41 / 19)\end{array}$ & $\begin{array}{c}\mathrm{T}: 62.07 \pm 8.88 \\
\mathrm{C}: 57.3 \pm 9.09\end{array}$ & $\begin{array}{l}1999 \text { WHO-ISH GMH and } \\
\text { Chinese Guidelines for the } \\
\text { Management of } \\
\text { Hypertension-2005 } \\
\text { (CGMH-2005) }\end{array}$ & Blood stasis (60) & Guangdong \\
\hline
\end{tabular}


TABle 1: Continued.

\begin{tabular}{|c|c|c|c|c|c|}
\hline Study ID & $\begin{array}{l}\text { Sample } \\
(\mathrm{M} / \mathrm{F})\end{array}$ & Age (years) & Diagnosis standard & $\begin{array}{l}\text { TCM syndrome differentiation } \\
\text { (number of patients) }\end{array}$ & $\begin{array}{l}\text { Region of } \\
\text { China }\end{array}$ \\
\hline $\begin{array}{l}\text { Liu et al. } 2009 \\
\text { [42] }\end{array}$ & $\begin{array}{c}60 \\
(36 / 24)\end{array}$ & $\begin{array}{l}\mathrm{T}: 53.87 \pm 5.92 \\
\mathrm{C}: 52.97 \pm 5.40\end{array}$ & $\begin{array}{l}\text { Chinese Guidelines for the } \\
\text { Management of } \\
\text { Hypertension-2005 } \\
\text { (CGMH-2005) }\end{array}$ & Phlegm-damp retention (60) & Shanghai \\
\hline $\begin{array}{l}\text { Wang et al. } \\
2012 \text { [43] }\end{array}$ & $\begin{array}{c}82 \\
(49 / 33)\end{array}$ & $60-75$ & 1999 WHO-ISH GMH & Liver-kidney yin deficiency (82) & Heilongjiang \\
\hline $\begin{array}{l}\text { Luo et al. } 2011 \\
\text { [44] }\end{array}$ & $\begin{array}{c}100 \\
(42 / 58)\end{array}$ & $36-81$ & $\begin{array}{l}\text { Hypertension diagnostic } \\
\text { criteria (unclear) }\end{array}$ & $\begin{array}{l}\text { Ascendant hyperactivity of liver yang (12), } \\
\text { yin deficiency with yang hyperactivity (60), } \\
\text { phlegm-damp retention (18), and kidney } \\
\text { deficiency (10) }\end{array}$ & Guangxi \\
\hline $\begin{array}{l}\text { Wang et al. } 2012 \\
\text { [45] }\end{array}$ & $\begin{array}{c}80 \\
(48 / 32)\end{array}$ & $68.05 \pm 5.41$ & 1999 WHO-ISH GMH & Liver-kidney yin deficiency (80) & Guangxi \\
\hline $\begin{array}{l}\text { Wang et al. } 2011 \\
\text { [46] }\end{array}$ & $\begin{array}{c}251 \\
(148 / 103)\end{array}$ & $55 \pm 19$ & 1999 WHO-ISH GMH & $\begin{array}{l}\text { Intense liver fire ( } 71 \text { ), yin deficiency with } \\
\text { yang hyperactivity (62), phlegm-damp } \\
\text { retention ( } 60) \text {, and deficiency of both yin } \\
\text { and yang (58) }\end{array}$ & Liaoning \\
\hline $\begin{array}{l}\text { Bai et al. } 2005 \\
\text { [47] }\end{array}$ & $\begin{array}{c}122 \\
(71 / 51)\end{array}$ & $\begin{array}{l}\text { T: } 44.7 \pm 11.6 \\
\text { C: } 46.2 \pm 9.5\end{array}$ & 1999 WHO-ISH GMH & $\begin{array}{l}\text { Ascendant hyperactivity of liver yang (35), } \\
\text { liver-kidney yin deficiency (18), } \\
\text { phlegm-damp retention (32), dual } \\
\text { deficiency of qi and yin (25), and stasis } \\
\text { blocking channels (12) }\end{array}$ & Hebei \\
\hline $\begin{array}{l}\text { Yang et al. } 2005 \\
\text { [48] }\end{array}$ & $\begin{array}{c}80 \\
(41 / 39)\end{array}$ & $\begin{array}{l}\text { M: } 51.28 \pm 6.96 \\
\text { F: } 52.71 \pm 6.57\end{array}$ & 1999 WHO-ISH GMH & Ascendant hyperactivity of liver yang (80) & Henan \\
\hline $\begin{array}{l}\text { Xia et al. } 2010 \\
{[49]}\end{array}$ & $\begin{array}{l}40 \\
\text { (M/F not } \\
\text { reported })\end{array}$ & $\begin{array}{l}\text { T: } 55.23 \pm 6.01 \\
\text { C: } 55.13 \pm 6.34\end{array}$ & 1999 WHO-ISH GMH & Ascendant hyperactivity of liver yang (40) & Gansu \\
\hline $\begin{array}{l}\text { Liu et al. } 2003 \\
\text { [50] }\end{array}$ & $\begin{array}{c}60 \\
(43 / 17)\end{array}$ & $45-73$ & $\begin{array}{l}\text { Chinese Guidelines for the } \\
\text { Management of } \\
\text { Hypertension-2009 } \\
\text { (CGMH-2009) }\end{array}$ & $\begin{array}{l}\text { Yang hyperactivity (29), phlegm turbidity } \\
\text { resistance (31) }\end{array}$ & Zhejiang \\
\hline $\begin{array}{l}\text { Yin and Liu } \\
2005[51]\end{array}$ & $\begin{array}{l}36 \\
\text { (M/F not } \\
\text { reported })\end{array}$ & $40.50 \pm 11.51$ & 1999 WHO-ISH GMH & Phlegm-damp retention $(36)$ & Jiangsu \\
\hline $\begin{array}{l}\text { Wu et al. } 2010 \\
{[52]}\end{array}$ & $\begin{array}{c}90 \\
(41 / 39)\end{array}$ & $32-78$ & 1999 WHO-ISH GMH & $\begin{array}{l}\text { Ascendant hyperactivity of liver yang and } \\
\text { blood stasis }(90)\end{array}$ & Hebei \\
\hline Deng 2008 [53] & $\begin{array}{c}60 \\
(45 / 15)\end{array}$ & $\begin{array}{l}\mathrm{T}: 61 \pm 4.12 \\
\mathrm{C}: 61 \pm 4.02\end{array}$ & 1999 WHO-ISH GMH & Qi deficiency with blood stasis (60) & Hebei \\
\hline $\begin{array}{l}\text { Wu and Xu } 2010 \\
\text { [54] }\end{array}$ & $\begin{array}{c}60 \\
(32 / 28)\end{array}$ & Not reported & 1999 WHO-ISH GMH & Dual deficiency of qi and yin (60) & Guizhou \\
\hline $\begin{array}{l}\text { Wang et al. } 2011 \\
\text { [55] }\end{array}$ & $\begin{array}{c}276 \\
170 / 106\end{array}$ & $\begin{array}{l}\text { M: } 53.4 \pm 21.1 \\
F: 55.6 \pm 17.3\end{array}$ & 1999 WHO-ISH GMH & $\begin{array}{l}\text { Wind-yang interference (22), stasis blocking } \\
\text { channels ( } 73) \text {, yin deficiency with yang } \\
\text { hyperactivity (134), and phlegm turbidity } \\
\text { resistance (47) }\end{array}$ & Guangxi \\
\hline $\begin{array}{l}\text { Wu et al. } 2010 \\
{[56]}\end{array}$ & $\begin{array}{c}156 \\
(79 / 77)\end{array}$ & $\begin{array}{l}\mathrm{T}: 48 \pm 6.9 \\
\mathrm{C}: 49 \pm 8.2\end{array}$ & $\begin{array}{l}\text { Hypertension diagnostic } \\
\text { criteria (unclear) }\end{array}$ & $\begin{array}{l}\text { Ascendant hyperactivity of liver yang (52), } \\
\text { yin deficiency with yang hyperactivity ( } 53) \text {, } \\
\text { and deficiency of both yin and yang (51) }\end{array}$ & Zhejiang \\
\hline $\begin{array}{l}\text { Fan and Liu } \\
2010 \text { [57] }\end{array}$ & $\begin{array}{c}395 \\
(203 / 192)\end{array}$ & $30-80$ & $\begin{array}{l}\text { Chinese Guidelines for the } \\
\text { Management of } \\
\text { Hypertension-2005 } \\
\text { (CGMH-2005) }\end{array}$ & $\begin{array}{l}\text { Qi deficiency with blood stasis (65), intense } \\
\text { liver fire (91), yin deficiency with yang } \\
\text { hyperactivity (63), phlegm-damp retention } \\
\text { (57), deficiency of both yin and yang (39), } \\
\text { and dual deficiency of qi and blood (18) }\end{array}$ & Beijing \\
\hline
\end{tabular}


TABLE 1: Continued.

\begin{tabular}{|c|c|c|c|c|c|}
\hline Study ID & $\begin{array}{l}\text { Sample } \\
(\mathrm{M} / \mathrm{F})\end{array}$ & Age (years) & Diagnosis standard & $\begin{array}{l}\text { TCM syndrome differentiation } \\
\text { (number of patients) }\end{array}$ & $\begin{array}{l}\text { Region of } \\
\text { China }\end{array}$ \\
\hline $\begin{array}{l}\text { Zhu et al. } 2009 \\
\text { [58] }\end{array}$ & $\begin{array}{c}54 \\
(30 / 24)\end{array}$ & $61.74 \pm 14.89$ & 1999 WHO-ISH GMH & $\begin{array}{l}\text { Kidney yang deficiency (24), kidney yin } \\
\text { deficiency ( } 30)\end{array}$ & Yunnan \\
\hline $\begin{array}{l}\text { Liu et al. } 2009 \\
\text { [59] }\end{array}$ & $\begin{array}{c}140 \\
(68 / 72)\end{array}$ & $34-79$ & $\begin{array}{l}\text { Chinese Guidelines for the } \\
\text { Management of } \\
\text { Hypertension-2005 } \\
\text { (CGMH-2005) }\end{array}$ & $\begin{array}{l}\text { Intense liver fire (16), yin deficiency with } \\
\text { yang hyperactivity (52), phlegm-damp } \\
\text { retention (41), and deficiency of both yin } \\
\text { and yang (31) }\end{array}$ & Guangxi \\
\hline $\begin{array}{l}\text { He et al. } 2013 \\
{[60]}\end{array}$ & $\begin{array}{c}230 \\
(65 / 165)\end{array}$ & $43-74$ & 1999 WHO-ISH GMH & $\begin{array}{l}\text { Intense liver fire (28), ascendant } \\
\text { hyperactivity of liver yang (148), and } \\
\text { liver-kidney yin deficiency (54) }\end{array}$ & Guangdong \\
\hline $\begin{array}{l}\text { Tang et al. } 2012 \\
\text { [61] }\end{array}$ & $\begin{array}{c}100 \\
(37 / 63)\end{array}$ & $55.1 \pm 6.2$ & 1999 WHO-ISH GMH & $\begin{array}{l}\text { Intense liver fire (19), yin deficiency with } \\
\text { yang hyperactivity (29), deficiency of both } \\
\text { yin and yang (20), and liver-kidney yin } \\
\text { deficiency (32) }\end{array}$ & Shanghai \\
\hline $\begin{array}{l}\text { Gong et al. } 2010 \\
\text { [62] }\end{array}$ & $\begin{array}{c}120 \\
(60 / 60)\end{array}$ & $\begin{array}{l}\text { T: } 55.38 \pm 8.01 \\
\text { C: } 56.80 \pm 8.58\end{array}$ & 1999 WHO-ISH GMH & $\begin{array}{l}\text { Intense liver fire (30), yin deficiency with } \\
\text { yang hyperactivity (30), phlegm-damp } \\
\text { retention (30), and deficiency of both yin } \\
\text { and yang (30) }\end{array}$ & Shandong \\
\hline $\begin{array}{l}\text { Zhang et al. } \\
2005 \text { [63] }\end{array}$ & $\begin{array}{c}60 \\
(32 / 28)\end{array}$ & $62.22 \pm 6.12$ & $\begin{array}{l}\text { Chinese Guidelines for the } \\
\text { Management of } \\
\text { Hypertension-2004 } \\
\text { (CGMH-2004) }\end{array}$ & $\begin{array}{l}\text { Intense liver fire (8), yin deficiency with } \\
\text { yang hyperactivity (28), phlegm-damp } \\
\text { retention (14), and deficiency of both yin } \\
\text { and yang (10) }\end{array}$ & Guangxi \\
\hline $\begin{array}{l}\text { Liu et al. } 2009 \\
\text { [64] }\end{array}$ & $\begin{array}{c}200 \\
(105 / 95)\end{array}$ & $\begin{array}{r}\text { M: } 61.88 \pm 11.91 \\
\text { F: } 63.07 \pm 12.45\end{array}$ & $\begin{array}{l}\text { Chinese Guidelines for the } \\
\text { Management of } \\
\text { Hypertension-2005 } \\
\text { (CGMH-2005) }\end{array}$ & $\begin{array}{l}\text { Intense liver fire (51), yin deficiency with } \\
\text { yang hyperactivity ( } 49) \text {, phlegm-damp } \\
\text { retention (50), and deficiency of both yin } \\
\text { and yang (50) }\end{array}$ & Guangxi \\
\hline Wang 2012 [65] & $\begin{array}{c}200 \\
(103 / 94)\end{array}$ & $46.4 \pm 15.46$ & 1999 WHO-ISH GMH & $\begin{array}{l}\text { Intense liver fire (96), yin deficiency with } \\
\text { yang hyperactivity (46), phlegm-damp } \\
\text { retention (18), and deficiency of both yin } \\
\text { and yang (37) }\end{array}$ & Shanxi \\
\hline $\begin{array}{l}\text { Yao and Huang } \\
2007[66]\end{array}$ & $\begin{array}{c}47 \\
(22 / 25)\end{array}$ & $66.00 \pm 12.35$ & $\begin{array}{l}\text { Chinese Guidelines for the } \\
\text { Management of } \\
\text { Hypertension-2004 } \\
\text { (CGMH-2004) }\end{array}$ & $\begin{array}{l}\text { Intense liver fire (12), yin deficiency with } \\
\text { yang hyperactivity (11), phlegm-damp } \\
\text { retention (12), and deficiency of both yin } \\
\text { and yang (12) }\end{array}$ & Tianjin \\
\hline $\begin{array}{l}\text { Guo et al. } 2002 \\
{[67]}\end{array}$ & $\begin{array}{c}120 \\
(62 / 58)\end{array}$ & $\begin{array}{l}\text { T: } 63.64 \pm 9.22 \\
\text { C: } 60.30 \pm 3.36\end{array}$ & $\begin{array}{l}\text { Chinese Guidelines for the } \\
\text { Management of } \\
\text { Hypertension-2004 } \\
\text { (CGMH-2004) }\end{array}$ & $\begin{array}{l}\text { Intense liver fire (30), yin deficiency with } \\
\text { yang hyperactivity (30), phlegm-damp } \\
\text { retention (30), and deficiency of both yin } \\
\text { and yang (30) }\end{array}$ & Anhui \\
\hline $\begin{array}{l}\text { Zhang et al. } 2011 \\
\text { [68] }\end{array}$ & $\begin{array}{c}320 \\
(135 / 185)\end{array}$ & $66.40 \pm 12.56$ & 2007 WHO-ISH GMH & $\begin{array}{l}\text { Intense liver fire (36), yin deficiency with } \\
\text { yang hyperactivity (101), phlegm-damp } \\
\text { retention (125), and deficiency of both yin } \\
\text { and yang (58) }\end{array}$ & Jiangsu \\
\hline $\begin{array}{l}\text { Liao et al. } 2010 \\
\text { [69] }\end{array}$ & $\begin{array}{c}23 \\
(14 / 9)\end{array}$ & $\begin{array}{l}\mathrm{T}: 65 \pm 5 \\
\mathrm{C}: 65 \pm 8\end{array}$ & 1999 WHO-ISH GMH & Blood stasis (23) & Fujian \\
\hline Xiong 2010 [70] & $\begin{array}{c}70 \\
(37 / 33)\end{array}$ & $53.06 \pm 8.62$ & $\begin{array}{l}\text { Hypertension diagnostic } \\
\text { criteria (unclear) }\end{array}$ & $\begin{array}{l}\text { Intense liver fire (13), yin deficiency with } \\
\text { yang hyperactivity (21), phlegm-damp } \\
\text { retention (25), and deficiency of both yin } \\
\text { and yang (11) }\end{array}$ & Heilongjiang \\
\hline $\begin{array}{l}\text { Jiang et al. } 2012 \\
\text { [71] }\end{array}$ & $\begin{array}{c}86 \\
(50 / 36)\end{array}$ & $36-81$ & $\begin{array}{l}\text { Hypertension diagnostic } \\
\text { criteria (unclear) }\end{array}$ & Yin deficiency with yang hyperactivity (86) & Guangdong \\
\hline $\begin{array}{l}\text { Huang and Wei } \\
2012[72]\end{array}$ & $\begin{array}{c}260 \\
(119 / 141)\end{array}$ & $65.56 \pm 8.42$ & $\begin{array}{l}\text { Chinese Guidelines for the } \\
\text { Management of } \\
\text { Hypertension-2010 } \\
\text { (CGMH-2010) }\end{array}$ & $\begin{array}{l}\text { Intense liver fire (56), yin deficiency with } \\
\text { yang hyperactivity ( } 77) \text {, phlegm-damp } \\
\text { retention ( } 73) \text {, and deficiency of both yin } \\
\text { and yang (54) }\end{array}$ & Beijing \\
\hline
\end{tabular}


TABLE 1: Continued.

\begin{tabular}{|c|c|c|c|c|c|}
\hline Study ID & $\begin{array}{l}\text { Sample } \\
(\mathrm{M} / \mathrm{F})\end{array}$ & Age (years) & Diagnosis standard & $\begin{array}{l}\text { TCM syndrome differentiation } \\
\text { (number of patients) }\end{array}$ & $\begin{array}{l}\text { Region of } \\
\text { China }\end{array}$ \\
\hline Lu 2004 [73] & $\begin{array}{c}138 \\
(97 / 41)\end{array}$ & $61.84 \pm 5.25$ & 1999 WHO-ISH GMH & $\begin{array}{l}\text { Intense liver fire (16), yin deficiency with } \\
\text { yang hyperactivity (43), phlegm-damp } \\
\text { retention (45), and deficiency of both yin } \\
\text { and yang (34) }\end{array}$ & Fujian \\
\hline $\begin{array}{l}\text { Sun and Wang } \\
2005[74]\end{array}$ & $\begin{array}{c}703 \\
(382 / 321)\end{array}$ & $50-79$ & $\begin{array}{l}\text { Chinese Guidelines for the } \\
\text { Management of } \\
\text { Hypertension-2005 } \\
\text { (CGMH-2005) }\end{array}$ & $\begin{array}{l}\text { Yin deficiency with yang hyperactivity (215), } \\
\text { phlegm-damp retention (83), deficiency of } \\
\text { both yin and yang (91), ascendant } \\
\text { hyperactivity of liver yang (135), liver-kidney } \\
\text { yin deficiency (92), yang deficiency (11), qi } \\
\text { deficiency (14), dual deficiency of qi and yin } \\
\text { (14), blood stasis (11), qi deficiency with } \\
\text { blood stasis (3), internal harassment of } \\
\text { phlegm-heat (22), internal harassment of } \\
\text { phlegm-heat and blood stasis (3), } \\
\text { liver-kidney yin deficiency and blood stasis } \\
\text { (2), internal harassment of phlegm-heat and } \\
\text { qi deficiency (1), deficiency of both yin and } \\
\text { yang and internal harassment of } \\
\text { phlegm-heat (1), liver-kidney yin deficiency } \\
\text { and phlegm-damp retention (1), yin } \\
\text { deficiency with yang hyperactivity and } \\
\text { blood stasis (1), ascendant hyperactivity of } \\
\text { liver yang and internal harassment of } \\
\text { phlegm-heat (1), ascendant hyperactivity of } \\
\text { liver yang and blood stasis (1), and } \\
\text { deficiency of both yin and yang and } \\
\text { phlegm-damp retention (1) }\end{array}$ & Guangdong \\
\hline $\begin{array}{l}\text { Xiang et al. } 2012 \\
{[75]}\end{array}$ & $\begin{array}{c}125 \\
(75 / 50)\end{array}$ & $55-72$ & $\begin{array}{l}\text { Hypertension diagnostic } \\
\text { criteria (unclear) }\end{array}$ & $\begin{array}{l}\text { Kidney deficiency and blood stasis (15), } \\
\text { internal stirring of liver wind (68), qi } \\
\text { deficiency with blood stasis (21), and } \\
\text { intermingled phlegm and blood stasis (21) }\end{array}$ & Guangdong \\
\hline Zhu 2009 [76] & $\begin{array}{c}97 \\
(41 / 56)\end{array}$ & $37-79$ & $\begin{array}{l}\text { Hypertension diagnostic } \\
\text { criteria (unclear) }\end{array}$ & $\begin{array}{l}\text { Ascendant hyperactivity of liver yang (13), } \\
\text { yin deficiency with yang hyperactivity ( } 59) \text {, } \\
\text { liver-kidney yin deficiency (16), and } \\
\text { deficiency of both yin and yang (21) }\end{array}$ & Shandong \\
\hline $\begin{array}{l}\text { Xu and Wang } \\
2009 \text { [77] }\end{array}$ & $\begin{array}{c}80 \\
(49 / 31)\end{array}$ & $40-83$ & 1999 WHO-ISH GMH & $\begin{array}{l}\text { Intense liver fire (18), yin deficiency with } \\
\text { yang hyperactivity (17), phlegm-damp } \\
\text { retention (35), and deficiency of both yin } \\
\text { and yang (10) }\end{array}$ & Xinjiang \\
\hline $\begin{array}{l}\text { Lin and Kang } \\
2012[78]\end{array}$ & $\begin{array}{c}69 \\
(37 / 32)\end{array}$ & $\begin{array}{l}\mathrm{T}: 53.48 \pm 10.02 \\
\mathrm{C}: 59.20 \pm 5.610\end{array}$ & $\begin{array}{l}\text { Chinese Guidelines for the } \\
\text { Management of } \\
\text { Hypertension-2005 } \\
\text { (CGMH-2005) }\end{array}$ & Liver-kidney yin deficiency (69) & Zhejiang \\
\hline $\begin{array}{l}\text { Feng et al. } 2013 \\
{[79]}\end{array}$ & $\begin{array}{c}60 \\
(60 \mathrm{M})\end{array}$ & $63.0 \pm 7.5$ & $\begin{array}{l}\text { Chinese Guidelines for the } \\
\text { Management of } \\
\text { Hypertension-2005 } \\
\text { (CGMH-2005) }\end{array}$ & Ascendant hyperactivity of liver yang (60) & Fujian \\
\hline $\begin{array}{l}\text { Yu and Xing } \\
2010[80]\end{array}$ & $\begin{array}{c}168 \\
(108 / 60)\end{array}$ & $\begin{array}{l}\text { T: } 58 \pm 12 \\
\text { C: } 54 \pm 12\end{array}$ & 1999 WHO-ISH GMH & $\begin{array}{l}\text { Intense liver fire (54), yin deficiency with } \\
\text { yang hyperactivity ( } 45) \text {, phlegm-damp } \\
\text { retention (36), and deficiency of both yin } \\
\text { and yang (33) }\end{array}$ & Beijing \\
\hline $\begin{array}{l}\text { Qiu et al. } 2011 \\
\text { [81] }\end{array}$ & $\begin{array}{c}170 \\
(122 / 48)\end{array}$ & $54 \pm 11.6$ & $\begin{array}{l}\text { Chinese Guidelines for the } \\
\text { Management of } \\
\text { Hypertension-2004 } \\
(\text { CGMH-2004) }\end{array}$ & $\begin{array}{l}\text { Intense liver fire (43), yin deficiency with } \\
\text { yang hyperactivity ( } 40) \text {, phlegm-damp } \\
\text { retention (38), and deficiency of both yin } \\
\text { and yang (49) }\end{array}$ & Beijing \\
\hline
\end{tabular}


TABle 1: Continued.

\begin{tabular}{|c|c|c|c|c|c|}
\hline Study ID & $\begin{array}{l}\text { Sample } \\
(\mathrm{M} / \mathrm{F})\end{array}$ & Age (years) & Diagnosis standard & $\begin{array}{l}\text { TCM syndrome differentiation } \\
\text { (number of patients) }\end{array}$ & $\begin{array}{c}\text { Region of } \\
\text { China }\end{array}$ \\
\hline $\begin{array}{l}\text { Wu and Xu } 2012 \\
{[82]}\end{array}$ & $\begin{array}{c}149 \\
(74 / 75)\end{array}$ & $61.22 \pm 9.36$ & $\begin{array}{l}\text { Hypertension diagnostic } \\
\text { criteria (unclear) }\end{array}$ & $\begin{array}{l}\text { Intense liver fire (48), yin deficiency with } \\
\text { yang hyperactivity (32), phlegm-damp } \\
\text { retention (49), and deficiency of both yin } \\
\text { and yang (20) }\end{array}$ & Hubei \\
\hline $\begin{array}{l}\text { Fang et al. } 2007 \\
{[83]}\end{array}$ & $\begin{array}{c}220 \\
(128 / 92)\end{array}$ & $34-73$ & 1999 WHO-ISH GMH & $\begin{array}{l}\text { Intense liver fire (98), yin deficiency with } \\
\text { yang hyperactivity (79), phlegm-damp } \\
\text { retention (19), and deficiency of both yin } \\
\text { and yang (24) }\end{array}$ & Gansu \\
\hline $\begin{array}{l}\text { Fang et al. } 2003 \\
{[84]}\end{array}$ & $\begin{array}{c}229 \\
(113 / 116)\end{array}$ & $>35$ & $\begin{array}{l}\text { Hypertension diagnostic } \\
\text { criteria (unclear) }\end{array}$ & $\begin{array}{l}\text { Liver-kidney yin deficiency (60), yin } \\
\text { deficiency with yang hyperactivity ( } 73) \text {, } \\
\text { phlegm-damp retention (85), and deficiency } \\
\text { of both yin and yang (11) }\end{array}$ & Hangzhou \\
\hline $\begin{array}{l}\text { Peng and Shi } \\
2010[85]\end{array}$ & $\begin{array}{c}122 \\
(57 / 65)\end{array}$ & $64.62 \pm 8.86$ & 1999 WHO-ISH GMH & $\begin{array}{l}\text { Qi deficiency with blood stasis (26), intense } \\
\text { liver fire (23), yin deficiency with yang } \\
\text { hyperactivity (26), phlegm-damp retention } \\
\text { (25), and deficiency of both yin and yang } \\
(22)\end{array}$ & Anhui \\
\hline $\begin{array}{l}\text { Yang et al. } 2004 \\
{[86]}\end{array}$ & $\begin{array}{c}151 \\
(110 / 41)\end{array}$ & Not reported & $\begin{array}{l}\text { Hypertension diagnostic } \\
\text { criteria (unclear) }\end{array}$ & Ascendant hyperactivity of liver yang (151) & Shandong \\
\hline $\begin{array}{l}\text { Shi et al. } 2013 \\
\text { [87] }\end{array}$ & $\begin{array}{c}60 \\
(29 / 31)\end{array}$ & $52.6 \pm 12.3$ & $\begin{array}{l}\text { Clinical research guiding } \\
\text { principles of new medicine } \\
\text { of Chinese traditional } \\
\text { medicine }\end{array}$ & Phlegm-damp retention $(60)$ & Zhejiang \\
\hline Han 2004 [88] & $\begin{array}{c}377 \\
(182 / 195)\end{array}$ & $20-60$ & $\begin{array}{l}\text { Chinese Guidelines for the } \\
\text { Management of } \\
\text { Hypertension-2005 } \\
\text { (CGMH-2005) }\end{array}$ & $\begin{array}{l}\text { Intense liver fire (108), yin deficiency with } \\
\text { yang hyperactivity (70), phlegm-damp } \\
\text { retention (154), and deficiency of both yin } \\
\text { and yang (45) }\end{array}$ & Anhui \\
\hline $\begin{array}{l}\text { Shen et al. } 2008 \\
\text { [89] }\end{array}$ & $\begin{array}{c}79 \\
(40 / 39)\end{array}$ & $\begin{array}{l}\text { T: } 51.70 \pm 4.53 \\
\text { C: } 51.67 \pm 4.36\end{array}$ & $\begin{array}{l}\text { Clinical research guiding } \\
\text { principles of new medicine } \\
\text { of Chinese traditional } \\
\text { medicine }\end{array}$ & $\begin{array}{l}\text { Phlegm and blood stasis resistance winding } \\
\text { and ascendant hyperactivity of liver yang } \\
\text { (79) }\end{array}$ & Guangzhou \\
\hline $\begin{array}{l}\text { Shen et al. } 2005 \\
{[90]}\end{array}$ & $\begin{array}{c}290 \\
(120 / 170)\end{array}$ & $66.2 \pm 1.37$ & $\begin{array}{l}\text { Chinese Guidelines for the } \\
\text { Management of } \\
\text { Hypertension-2004 } \\
\text { (CGMH-2004) }\end{array}$ & $\begin{array}{l}\text { Intense liver fire (34), yin deficiency with } \\
\text { yang hyperactivity (99), phlegm-damp } \\
\text { retention (114), and deficiency of both yin } \\
\text { and yang (43) }\end{array}$ & Jiangsu \\
\hline $\begin{array}{l}\text { Liu et al. } 2009 \\
\text { [91] }\end{array}$ & $\begin{array}{c}240 \\
(120 / 120)\end{array}$ & $18-65$ & $\begin{array}{l}\text { Hypertension diagnostic } \\
\text { criteria (unclear) }\end{array}$ & Intense liver fire (240) & Anhui \\
\hline $\begin{array}{l}\text { Lu et al. } 2011 \\
{[92]}\end{array}$ & $\begin{array}{c}80 \\
(56 / 24)\end{array}$ & $\begin{array}{l}\mathrm{T}: 66.07 \pm 7.15 \\
\mathrm{C}: 67.10 \pm 7.32\end{array}$ & 1999 WHO-ISH GMH & Blood stasis (80) & Guangxi \\
\hline $\begin{array}{l}\text { Guo et al. } 2006 \\
{[93]}\end{array}$ & $\begin{array}{c}60 \\
(30 / 30)\end{array}$ & Not reported & $\begin{array}{l}\text { Chinese Guidelines for the } \\
\text { Management of } \\
\text { Hypertension-2010 } \\
\text { (CGMH-2010) }\end{array}$ & Yin deficiency with yang hyperactivity (60) & Fujian \\
\hline $\begin{array}{l}\text { Zhang et al. } 2012 \\
\text { [94] }\end{array}$ & $\begin{array}{c}140 \\
(83 / 57)\end{array}$ & $56 \pm 10$ & $\begin{array}{l}\text { Chinese Guidelines for the } \\
\text { Management of } \\
\text { Hypertension-2010 } \\
\text { (CGMH-2010) }\end{array}$ & $\begin{array}{l}\text { Ascendant hyperactivity of liver yang (28), } \\
\text { yin deficiency with yang hyperactivity (39), } \\
\text { phlegm-damp retention (45), and deficiency } \\
\text { of both yin and yang (28) }\end{array}$ & Hebei \\
\hline $\begin{array}{l}\text { Dong et al. } 2010 \\
\text { [95] }\end{array}$ & $\begin{array}{c}166 \\
(106 / 60)\end{array}$ & $63-82$ & $\begin{array}{l}\text { Hypertension diagnostic } \\
\text { criteria (unclear) }\end{array}$ & $\begin{array}{l}\text { Kidney yin deficiency and wind-phlegm } \\
\text { (166) }\end{array}$ & Sichuan \\
\hline
\end{tabular}

(9.93\%). Among them, there were 879 cases of kidney yin deficiency (97.34\%) and 24 cases of kidney yang deficiency (2.66\%). There were 399 cases of spleen syndromes $(4.39 \%)$, all of which were spleen qi deficiency.
3.5. Combining Forms of Syndrome Elements of EH. We found that 13,272 cases of hypertension contained 33 syndrome types. According to the definition of syndrome elements, all syndromes were divided into four types: single factor, 
TABLE 2: Number of papers and cases in region.

\begin{tabular}{|c|c|c|c|c|c|}
\hline Region (China) & Provinces & Papers (pieces) & Cases & Male & Female \\
\hline \multirow{4}{*}{ North China } & Hebei & 5 & 552 & 323 & 229 \\
\hline & Beijing & 6 & 1374 & 718 & 656 \\
\hline & Inner Mongolia & 1 & 53 & 16 & 37 \\
\hline & Tianjin & 2 & 247 & 131 & 116 \\
\hline \multirow{2}{*}{ Northeast } & Liaoning & 2 & 646 & 376 & 270 \\
\hline & Heilongjiang & 2 & 152 & 86 & 66 \\
\hline \multirow{3}{*}{ Northwest } & Xinjiang & 2 & 230 & 124 & 106 \\
\hline & Shanxi & 1 & 197 & 103 & 94 \\
\hline & Gansu & 2 & 260 & 148 & 112 \\
\hline \multirow{3}{*}{ Central China } & Henan & 2 & 200 & 101 & 99 \\
\hline & Hubei & 1 & 149 & 74 & 75 \\
\hline & Hunan & 1 & 120 & 64 & 56 \\
\hline \multirow{7}{*}{ East China } & Shandong & 3 & 368 & 211 & 157 \\
\hline & Jiangsu & 10 & 3004 & 1489 & 1515 \\
\hline & Anhui & 4 & 859 & 421 & 438 \\
\hline & Zhejiang & 6 & 655 & 354 & 301 \\
\hline & Fujian & 6 & 466 & 307 & 159 \\
\hline & Jiangxi & 1 & 112 & 83 & 29 \\
\hline & Shanghai & 3 & 338 & 154 & 184 \\
\hline \multirow{2}{*}{ South China } & Guangdong & 12 & 1972 & 1047 & 925 \\
\hline & Guangxi & 7 & 936 & 519 & 417 \\
\hline \multirow{3}{*}{ Southwest China } & Yunnan & 1 & 54 & 30 & 24 \\
\hline & Guizhou & 2 & 162 & 90 & 72 \\
\hline & Sichuan & 1 & 166 & 106 & 60 \\
\hline \multicolumn{2}{|c|}{ Total } & 83 & 13272 & 7075 & 6197 \\
\hline
\end{tabular}

TABle 3: Syndrome elements of 13,272 patients with essential hypertension.

\begin{tabular}{lcc}
\hline Syndrome factors & Frequency & Percentage (\%) \\
\hline Yin deficiency & 5554 & 26.27 \\
Yang hyperactivity & 4033 & 19.08 \\
Phlegm turbidity & 2892 & 13.68 \\
Internal fire & 2793 & 13.21 \\
Internal dampness & 2333 & 11.04 \\
Yang deficiency & 1668 & 7.89 \\
Blood stasis & 1027 & 4.86 \\
Qi deficiency & 380 & 1.80 \\
Internal wind & 256 & 1.21 \\
Qi stagnation & 164 & 0.78 \\
Blood deficiency & 38 & 0.18 \\
\hline
\end{tabular}

two-factor, three-factor, and four-factor syndromes. The statistics of the combined forms of syndrome and their frequency (proportion more than 1\%) are shown in Table 5. Internal fire is the most common in the single factor group, while yin deficiency with yang hyperactivity is the most common in the two-factor group. From highest to lowest frequency in the two-factor group are phlegm-damp retention, deficiency of both yin and yang, Liver-kidney
TABLE 4: Targets of syndrome elements.

\begin{tabular}{lc}
\hline Target & Percentage (\%) \\
\hline Liver & $7789(85.68)$ \\
Kidney & $903(9.93)$ \\
Spleen & $399(4.39)$ \\
\hline Total & 100 \\
\hline
\end{tabular}

yin deficiency, dual deficiency of qi and yin, qi stagnation and blood stasis, and qi deficiency with blood stasis. The syndrome, yin deficiency and wind-phlegm, is the most common in the three-factor category. There were no fourfactor combinations that reached a frequency of greater than $1 \%$.

\section{Discussion and Perspectives}

4.1. Pathogenesis of Hypertension. Syndrome elements are the expression of pathogenesis of a disease [36]. According to the statistical results of syndrome elements, pathogenesis of $\mathrm{EH}$ can be summarized as simultaneous insufficiency and excess. Deficiency syndrome included yin deficiency, yang deficiency, qi deficiency, and blood deficiency. Excess 
TABLE 5: Combined syndrome forms.

\begin{tabular}{llcc}
\hline Combination Class & Combination Forms & Frequency & Percentage (\%) \\
\hline \multirow{3}{*}{ Single-factor } & Internal fire & 2765 & 20.98 \\
& Yang hyperactivity & 875 & 6.64 \\
& Blood stasis & 398 & 3.02 \\
& Phlegm turbidity & 149 & 1.13 \\
\hline & Yin deficiency with yang hyperactivity & 3059 & 23.21 \\
& Phlegm-damp retention & 2508 & 19.03 \\
Two-factor & Deficiency of both yin and yang & 1605 & 12.18 \\
& Liver-kidney yin deficiency & 543 & 4.12 \\
& Dual deficiency of qi and yin & 189 & 1.43 \\
& Qi stagnation and blood stasis & 164 & 1.24 \\
\hline Three-factor & Qi deficiency with blood stasis & 138 & 1.05 \\
\hline & Yin deficiency and wind-phlegm & 166 & 1.26 \\
\hline
\end{tabular}
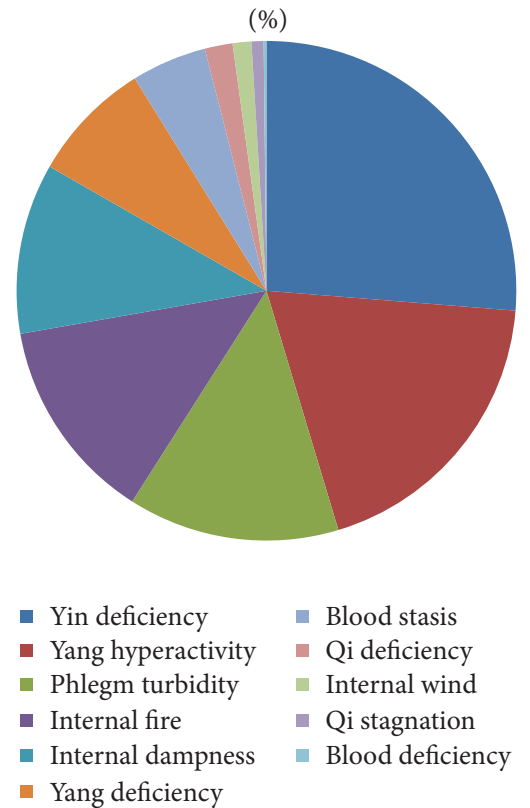

Figure 2: Percentage of syndrome factors.

syndrome included blood stasis, phlegm turbidity, qi stagnation, yang hyperactivity, internal fire, internal dampness, and internal wind. Among them, yin deficiency was the most common, followed by yang hyperactivity. Other elements, included in descending order, were phlegm turbidity, internal fire, internal dampness, yang deficiency, blood stasis, qi deficiency, and internal wind. The main disease location is the liver, which is closely related to the kidney and spleen.

4.2. Characteristics of Combined Syndrome Elements of EH. The combined forms of syndrome elements of hypertension have certain characteristics according to the literature, summarized as follows. (1) The combined forms of syndrome elements of hypertension have three forms, single-factor, two-factor, and three-factor forms. (2) Excess syndromes are more common than deficiency syndromes for singlefactor syndromes, with internal fire, yang hyperactivity, blood stasis, and phlegm turbidity as the main syndrome factors. (3) Deficiency syndrome and excess syndrome was the most common two-factor syndrome, followed by excess syndrome and excess syndrome and deficiency syndrome and deficiency syndrome, respectively. (4) Syndrome of yin deficiency and wind-phlegm was the most common threefactor syndrome.

4.3. Implications for Instructing Clinical Application. The discovery of distributing characteristics of syndrome elements is conducive to instructing clinical application. Several Chinese herbs and classical formulas can lower BP and improve symptoms according to syndrome differentiation (Table 6). First, when aiming to cure internal fire syndrome, use Huanglian Jie Du Tang (detoxicant decoction of Coptis) to clear heat and toxins of the liver [35]. Chinese herbs such as Xiakucao (Prunella vulgaris L.) [123], Huanglian (Coptis chinensis) [124], Huangqin (Scutellaria baicalensis Georgi), Huang-bai (Phellodendron bark), and Zhizi (Gardenia) [125] can lower BP. Second, when aiming to cure yin deficiency with yang hyperactivity, use Tianma Gouteng Yin (decoction of Gastrodia and Uncaria), a famous prescription noted in Za Bing Zheng Zhi Xin Yi (New Meanings in Syndrome and Therapy of Miscellaneous Diseases). Chinese herbs such as Tianma (Gastrodia) [126] and Gouteng (Uncaria) [127] could suppress liver yang hyperactivity. Niuxi (Achyranthes root) [128] and Duzhong (Eucommia ulmoides) [129-131] had antihypertensive effects by nourishing the kidney. Third, when aiming to cure phlegm-damp retention, use Wuling powder [132], Zexie Tang (decoction of Alisma) [133], and Wendantang jiawei decoction (modified decoction for clearing away gallbladder heat). In addition, when aiming at wind-phlegm syndrome, use Banxia Baizhu Tianma Tang (decoction of Pinellia ternata, Atractylodes macrocephala, and Gastrodia elata) to calm the liver, strengthen the spleen, remove dampness, and reduce phlegm [35]. Chinese herbs such as Zexie (Alisma), Fuling (Poria cocos) [134], Zhuling 


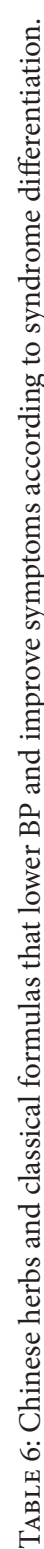

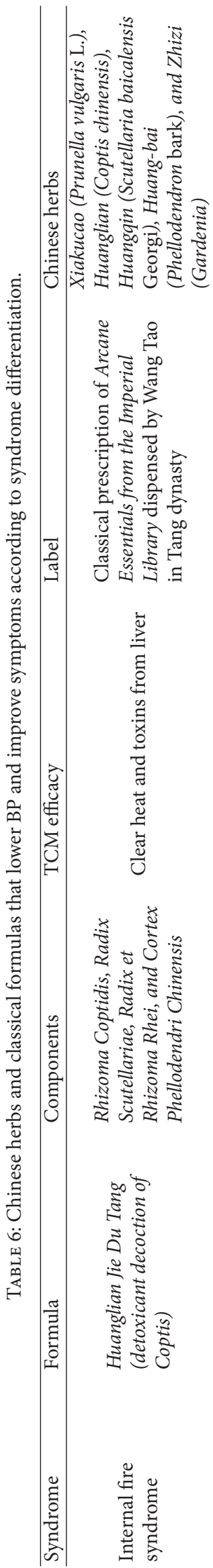

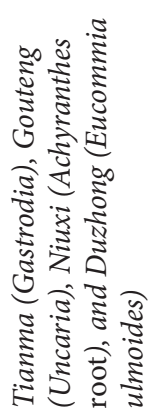

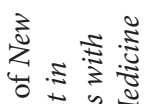

ป ङे

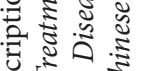

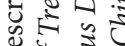

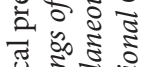

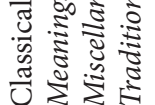

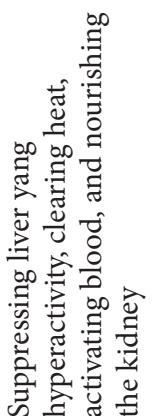

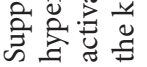
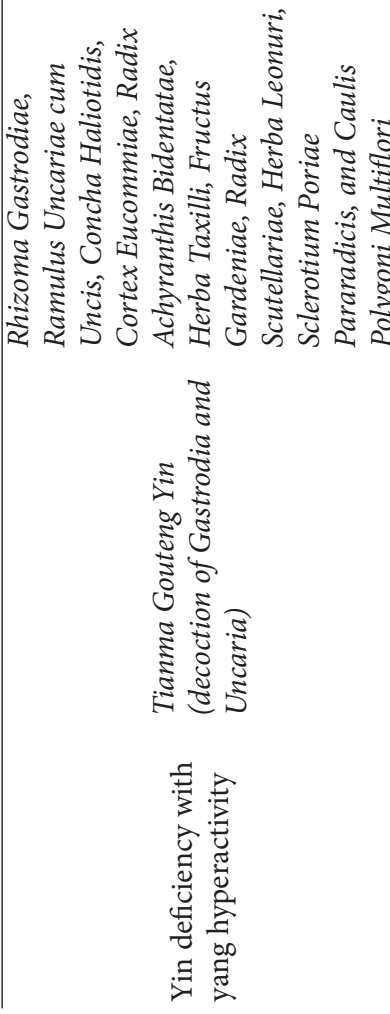
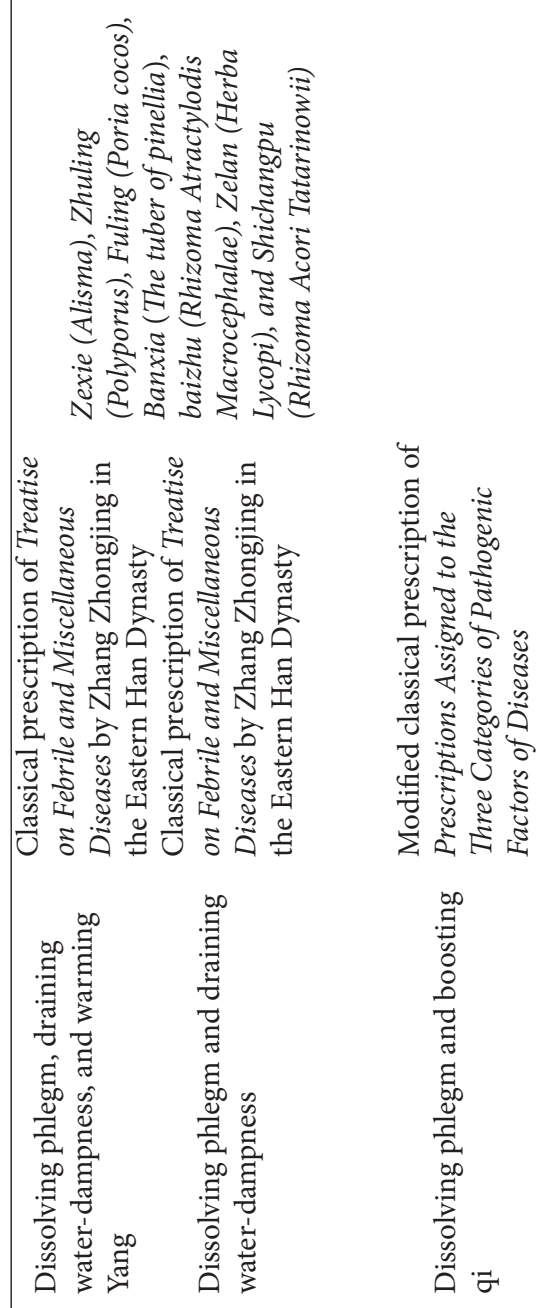

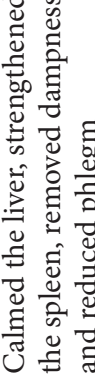

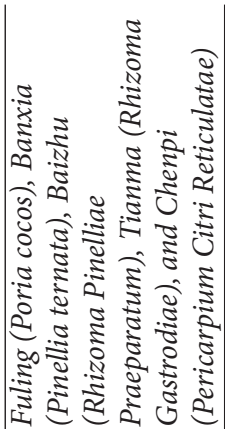

苛

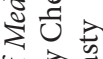

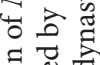
휴 क्षे \& $: \exists$

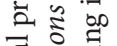

.

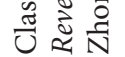

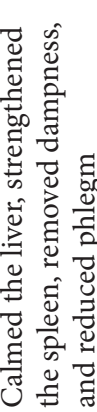

₹

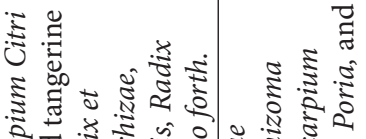

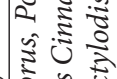
₹

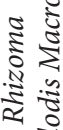

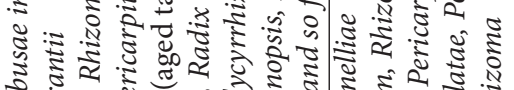

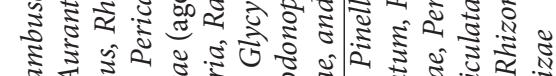

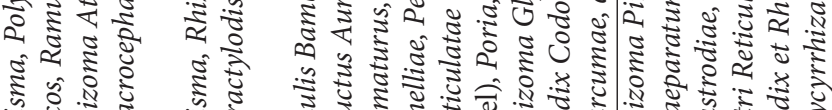

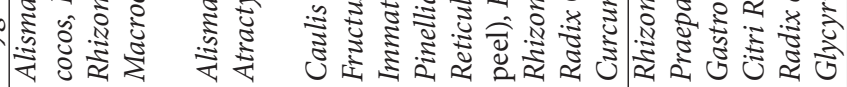
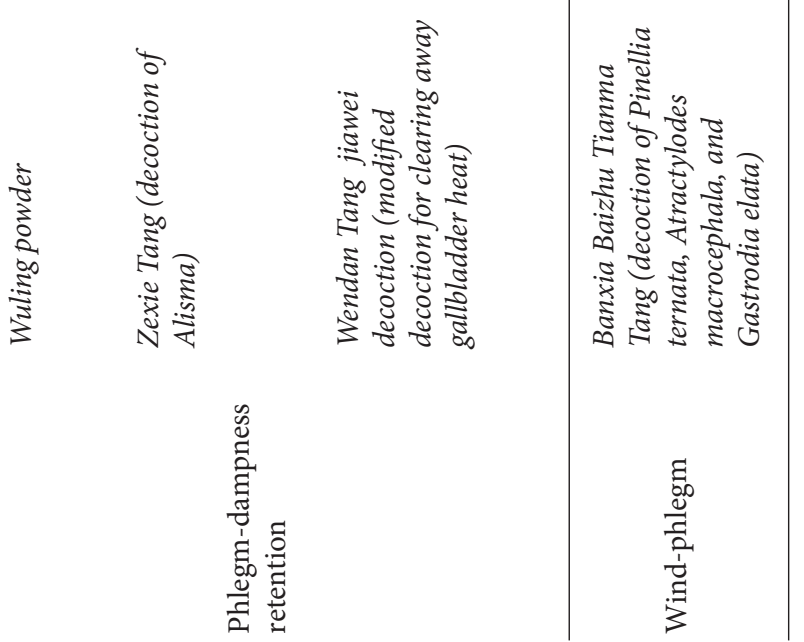


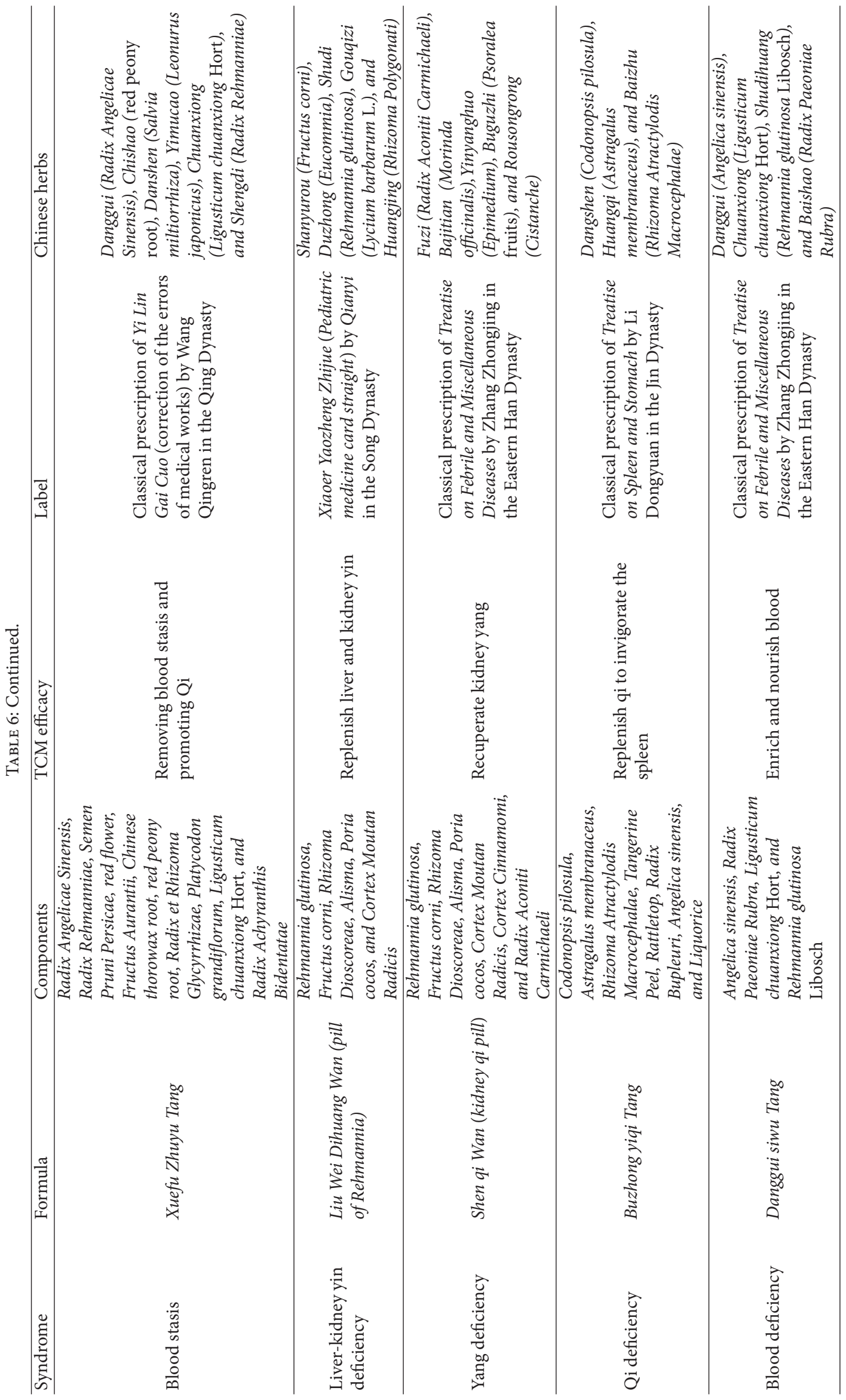


(Polyporus) [135], and Banxia (The tuber of pinellia) [136] could effectively reduce BP as well. Fourthly, to remove blood stasis, use Xuefu Zhuyu Tang, a famous classical prescription recorded in Yi Lin Gai Cuo (Correction of the Errors of Medical Works) by Wang Qingren in the Qing Dynasty. It is effective in removing blood stasis and promoting Qi. Herbs such as Chishao (red peony root) [137], Danshen (Salvia miltiorrhiza) [138], Yimucao (Leonurus japonicus), and Chuanxiong (Ligusticum chuanxiong Hort) [139] could also lower BP. When aiming to remove qi stagnation and blood stasis, use herbs to promote qi circulation by taking herbs to remove blood stasis. Herbs that promote qi circulation include Chaihu (Chinese thorowax root) [137], Cangzhu (Rhizoma Atractylodis), and Zhiqiao (Fructus Aurantii). Finally, deficiency syndromes including liver-kidney yin deficiency, yang deficiency, qi deficiency, and blood deficiency are common in hypertension. When curing liver-kidney yin deficiency, use Liu Wei Dihuang Wan (pill of Rehmannia) [23]. Liu Wei Dihuang Wan was recorded in Xiaoer Yaozheng Zhijue (Pediatric Medicine Card Straight) by Qianyi in the Song Dynasty, and it can replenish liver and kidney yin. When treating yang deficiency, use Shen qi Wan (kidney qi pill) to recuperate kidney yang. When aiming to treat qi deficiency, use Huangqi (Astragalus membranaceus) [140-142] and Baizhu (Rhizoma Atractylodis Macrocephalae). When aiming to treat blood deficiency, use Danggui (Angelica sinensis) [143], Shengdihuang (Radix Rehmanniae), Chuanxiong (Ligusticum chuanxiong Hort) [144], and Baishao (Radix Paeoniae Rubra).

In summary, the syndrome elements of hypertension are limited and are combined into syndromes. Single and the combined syndrome elements of hypertension are the basis of syndrome differentiation for $\mathrm{EH}$ and the key to the standardization of this syndrome. In this paper, we retrospectively confirmed the validity and reliability of the theory of syndrome elements and the combined forms of syndrome elements of hypertension. This study can provide new ideas and methods for the treatment of hypertension by syndrome differentiation, and has laid a foundation for researching syndrome standardization of hypertension.

\section{Conflict of Interests}

The authors declare that there is no conflict of interests regarding the publication of this paper.

\section{Authors' Contribution}

Jie Wang and Xingjiang Xiong contributed equally in this paper.

\section{References}

[1] M. Slama, D. Susic, and E. D. Frohlich, "Prevention of hypertension," Current Opinion in Cardiology, vol. 17, no. 5, pp. 531-536, 2002.

[2] D. A. Calhoun, M. A. Zaman, and M. K. Nishizaka, "Resistant hypertension," Current Hypertension Reports, vol. 4, no. 3, pp. 221-228, 2002.
[3] V. L. Roger, D. M. Lloyd-Jones, A. S. Go et al., "Heart disease and stroke statistics 2011 update: a report from the American Heart Association," Circulation, vol. 123, pp. el18-e209, 2011.

[4] P. M. Kearney, M. Whelton, K. Reynolds, P. Muntner, P. K. Whelton, and J. He, "Global burden of hypertension: analysis of worldwide data," Lancet, vol. 365, no. 9455, pp. 217-223, 2005.

[5] D. Lloyd-Jones, R. Adams, M. Carnethon et al., "Heart disease and stroke statistics-2009 update: a report from the American Heart Association Statistics Committee and Stroke Statistics Subcommittee," Circulation, vol. 119, no. 3, pp. 480-486, 2009.

[6] D. Su and L. Li, "Trends in the use of complementary and alternative medicine in the United States: 2002-2007," Journal of Health Care for the Poor and Underserved, vol. 22, no. 1, pp. 296-310, 2011.

[7] S. M. Ching, Z. A. Zakaria, F. Paimin et al., "Complementary alternative medicine use among patients with type 2 diabetes mellitus in the primary care setting: a cross-sectional study in Malaysia," BMC Complementary and Alternative Medicine, vol. 13, article 148, 2013.

[8] C. Hawk, H. Ndetan, and M. W. Evans, "Potential role of complementary and alternative health care providers in chronic disease prevention and health promotion: an analysis of National Health Interview Survey data," Preventive Medicine, vol. 54, no. 1, pp. 18-22, 2012.

[9] X. J. Xiong, X. C. Yang, W. Liu et al., "Banxia baizhu tianma decoction for essential hypertension: a systematic review of randomized controlled trials," Evidence-Based Complementary and Alternative Medicine, vol. 2012, Article ID 271462, 10 pages, 2012.

[10] X. J. Xiong, X. C. Yang, B. Feng et al., “Zhen gan xi feng decoction, a traditional Chinese herbal formula, for the treatment of essential hypertension: a systematic review of randomized controlled trials," Evidence-Based Complementary and Alternative Medicine, vol. 2013, Article ID 982380, 9 pages, 2013.

[11] J. Wang, X. C. Yang, B. Feng et al., "Is yangxue qingnao granule combined with antihypertensive drugs, a new integrative medicine therapy, more effective than antihypertensive therapy alone in treating essential hypertension?" Evidence-Based Complementary and Alternative Medicine, vol. 2013, Article ID 540613, 8 pages, 2013.

[12] W. Y. Tam, P. Chook, M. Qiao et al., "Cardiovascular protective effects of adjunctive alternative medicine (Salvia miltiorrhiza and Pueraria lobata) in high-risk hypertension," Evidence-Based Complementary and Alternative Medicine, vol. 2013, Article ID 132912, 8 pages, 2013.

[13] H. Xu and K. J. Chen, "Complementary and alternative medicine: is it possible to be main stream?" Chinese Journal of Integrative Medicine, vol. 18, pp. 403-404, 2012.

[14] A. S. Ferreira and A. J. Lopes, "Chinese medicine pattern differentiation and its implications for clinical practice," Chinese Journal of Integrative Medicine, vol. 17, no. 11, pp. 818-823, 2011.

[15] J. Wang, X. J. Xiong, and W. Liu, "Yoga for essential hypertension: a systemic review," PloS ONE, vol. 8, no. 10, Article ID e76357, 2013.

[16] M. S. Lee, H. J. Lim, and M. S. Lee, "Impact of qigong exercise on self-efficacy and other cognitive perceptual variables in patients with essential hypertension," Journal of Alternative and Complementary Medicine, vol. 10, no. 4, pp. 675-680, 2004.

[17] J. Wang, B. Feng, X. C. Yang et al., "Tai Chi for Essential Hypertension," Evidence-Based Complementary and Alternative Medicine, vol. 2013, Article ID 215254, 10 pages, 2013. 
[18] J. Wang and X. J. Xiong, "Outcome measures of Chinese herbal medicine for hypertension: an overview of systematic reviews," Evidence-Based Complementary and Alternative Medicine,, vol. 2012, Article ID 697237, 7 pages, 2012.

[19] X. J. Xiong, X. C. Yang, Y. M. Liu et al., "Chinese herbal formulas for treating hypertension in traditional Chinese medicine: perspective of modern science," Hypertension Research, vol. 36, pp. 570-579, 2013.

[20] J. Wang, B. Feng, X. C. Yang et al., “Tianma gouteng yin as adjunctive treatment for essential hypertension: a systematic review of randomized controlled trials," Evidence-Based Complementary and Alternative Medicine, vol. 2013, Article ID 706125, 18 pages, 2013.

[21] J. Wang, B. Feng, X. C. Yang, W. Liu, and X. Xiong, "Chinese herbal medicine for the treatment of prehypertension," Evidence-Based Complementary and Alternative Medicine, vol. 2013, Article ID 493521, 9 pages, 2013.

[22] K. J. Chen, "Mao ZD and integrative medicine," Zhongguo Zhong Xi Yi JieHe Za Zhi, vol. 13, no. 2, pp. 711-713, 1993.

[23] J. Wang, K. W. Yao, X. C. Yang et al., "Chinese patent medicine liu wei di huang wan combined with antihypertensive drugs, a new integrative medicine therapy, for the treatment of essential hypertension: a systematic review of randomized controlled trials," Evidence-Based Complementary and Alternative Medicine, vol. 2012, Article ID 714805, 7 pages, 2012.

[24] J. Wang, B. Feng, and X. J. Xiong, "Chinese herbal medicine for the treatment of obesity-related hypertension," Evidence-Based Complementary and Alternative Medicine, vol. 2013, Article ID 757540, 11 pages, 2013.

[25] J. Wang and X. J. Xiong, "Current situation and perspectives of clinical study in integrative medicine in China," Evidence-Based Complementary and Alternative Medicine, vol. 2012, Article ID 268542, 11 pages, 2012.

[26] K. J. Chen, Y. R. Jiang, and Y. H. Xie, "Past and present of combination of disease differentiation and syndrome differentiation," Zhongguo Zhong Xi Yi Jie He Za Zhi, vol. 31, no. 4, pp. 437-443, 2011.

[27] H. Xu and K. J. Chen, "Integrating traditional medicine with biomedicine towards a patient-centered healthcare system," Chinese Journal of Integrative Medicine, vol. 17, no. 2, pp. 83-84, 2011.

[28] S. L. Chen, X. Y. Liu, W. M. Xu, W. Y. Mei, and X. L. Chen, "Clinical study of western medicine combined with Chinese medicine based on syndrome differentiation in the patients with polarized hypertension," Chinese Journal of Integrative Medicine, vol. 18, no. 10, pp. 746-751, 2012.

[29] L. Liu, E. L. H. Leung, and X. Tian, "Perspective: the clinical trial barriers," Nature, vol. 480, 7378, p. S100, 2011.

[30] G. Dobos and I. Tao, "The model of Western Integrative Medicine: the role of Chinese medicine," Chinese Journal of Integrative Medicine, vol. 17, no. 1, pp. 11-20, 2011.

[31] X. J. Xiong, F. Y. Chu, H. X. Li, and Q. Y. He, "Clinical application of the TCM classic formulae for treating chronic bronchitis," Journal of Traditional Chinese Medicine, vol. 31, no. 1, pp. 69-72, 2011.

[32] J. Wang and X. Xiong, "Control strategy on hypertension in Chinese medicine," Evidence-Based Complementary and Alternative Medicine, vol. 2012, Article ID 284847, 6 pages, 2012.

[33] J. Wang, X. J. Xiong, G. Y. Yang et al., "Chinese herbal medicine qi ju di huang wan for the treatment of essential hypertension: a systematic review of randomized controlled trials," EvidenceBased Complementary and Alternative Medicine, vol. 2013, Article ID 262685, 10 pages, 2013.

[34] P. Tian, "Convergence: where West meets East," Nature, vol. 480, supplement 7378, pp. S84-S86, 2011.

[35] J. Wang and X. Xiong, "Control strategy on hypertension in Chinese medicine," Evidence-Based Complementary and Alternative Medicine, vol. 2012, Article ID 284847, 6 pages, 2012.

[36] J. Wang, P. Q. Wang, and X. J. Xiong, "Current situation and reunderstanding of syndrome and formula syndrome in Chinese medicine," Internal Medicine, vol. 2, no. 3, Article ID 1000113, 2012.

[37] H. Xu and K. Chen, "Integrative medicine: the experience from China," Journal of Alternative and Complementary Medicine, vol. 14, no. 1, pp. 3-7, 2008.

[38] F. Cheung, “TCM: made in China," Nature, vol. 480, no. 7378, pp. S82-S83, 2011.

[39] X. J. Xiong, X. C. Yang, W. Liu et al., “Trends in the treatment of hypertension from the perspective of traditional Chinese medicine," Evidence-Based Complementary and Alternative Medicine, vol. 2013, Article ID 275279, 13 pages, 2013.

[40] A. P. Lu, H. W. Jia, C. Xiao, and Q. P. Lu, "Theory of traditional Chinese medicine and therapeutic method of diseases," World Journal of Gastroenterology, vol. 10, no. 13, pp. 1854-1856, 2004.

[41] Q. G. Zhao, H. Li, H. M. Yang et al., "Difference of urine protein during the stage of early renal damage in elderly primary hypertensive patients with different yraditional Chinese Medical syndrome types: an analysis of 122 cases," Journal of Chinese Medicine, vol. 44, no. 7, pp. 25-27, 2012.

[42] F. M. Liu, X. H. Chen, W. Q. Du et al., "Correlativity between ACE Gene I/D polymorphism and TCM syndromes of primary hypertension," Shanghai Journal of Traditional Chinese Medicine, vol. 43, no. 11, pp. 33-35, 2009.

[43] X. Y. Wang, Z. Q. Xie, and L. L. Ji, "Clinical research of amlodipine combined Xingqi huoxue decoction on 99 elderly patients with qi stagnation and bood stasis hypertension," Chinese Medicine Modern Distance Education of China, vol. 10, no. 13, pp. 81-82, 2012.

[44] F. H. Luo, G. H. Li, Q. L. Li et al., "Clinical research on syndrome differentiation of point application in the treatment of primary hypertension," China Medical Herald, vol. 8, no. 21, pp. 108-112, 2011.

[45] C. H. Wang, Y. Gao, J. Z. Chen et al., "Effect of syndrome differentiation and treatment combined alisma decoction on traditional Chinese medicine symptom integral of hypertension," Global Traditional Chinese Medicine, vol. 5, no. 7, pp. 538540, 2012.

[46] Y. S. Wang, Z. H. Lin, and H. C. Chen, "Clinical research on the treatment of early hypertensive renal damage by tonifying kidney and invigorating the circulation of blood," Traditional Chinese Medicine Journal, vol. 10, no. 5, pp. 52-54, 2011.

[47] C. J. Bai, Y. Zhou, L. Wang, D. L. Zhang, and Y. Yang, "Delamination of cardiovascular risk factor, staging and grading of hypertension and the changing characteristics of blood lipids and hemorheological indexes in hypertensive patients with different syndromes of traditional Chinese medicine," Chinese Journal of Clinical Rehabilitation, vol. 9, no. 23, pp. 145-147, 2005.

[48] L. J. Yang, L. Yao, A. F. Liu et al., "Relation study between erythrocyte immunological function and serum SOD activity of hypertension patients with different typing," Modern Journal of Integrated Traditional Chinese and Western Medicine, vol. 14, no. 13, pp. 1676-1677, 2005. 
[49] C. X. Xia, Q. Y. Yang, H. J. Zhu et al., "Correlation study on different TCM syndromes of primary hypertension and NO/NOS system, ET21, left ventricular mass index (BMI)," Chinese Journal of Integrative Medicine on Cardio/Cerebrovascular Disease, vol. 8, no. 1, pp. 32-33, 2010.

[50] Z. Y. Liu, H. L. Wu, Y. Luo et al., "The effect of shenmai injection dealing with insufficient relaxation of left ventricle caused by hypertension," Chinese Journal of Chinese Medicine Information, vol. 10, no. 4, pp. 16-18, 2003.

[51] W. H. Yin and J. L. Liu, "Clinical observation on treatment of 53 cases with essential hypertension by deficiency syndrome," Inner Mongolia Medical Journal, vol. 37, no. 6, pp. 536-537, 2005.

[52] R. Wu, F. D. Zhao, and S. J. Yu, "Research of different type of traditional medical syndromes on hypertension disease with regularity of blood pressure variability," Chinese Journal of Chinese Medicine Emergency, vol. 19, no. 3, pp. 443-445, 2010.

[53] H. F. Deng, "Analysis of effect on compound danshen dropping pill compared with sheng tong ping in patients with hypertension," Asia-Pacific Traditional Medicine, vol. 4, no. 10, pp. 46-48, 2008.

[54] L. Q. Wu and F. Q. Xu, "Study on the relationship between type of syndrome of high-risk hypertensive patients and target organ damage and the risk factors of cardiovascular events," Chinese Journal of Integrative Medicine on Cardio/Cerebrovascular Disease, vol. 8, no. 12, pp. 1409-1410, 2010.

[55] W. Wang, F. Q. Li, and Y. P. Li, "Study on the relationship between Syndrome differentiation type of hypertension and Angiotensin original gene M 235T and T 174M polymorphisms," Journal of Zhejiang Traditional Chinese Medicine, vol. 46, no. 9, pp. 628-629, 2011.

[56] D. M. Wu, X. H. Chen, and F. M. Liu, "Studying of relationship between arteriosclerosis of essential hypertension and TCM syndromes," Journal of Chinese Medicine in Inner Mongolia, vol. 3, pp. 1-2, 2010.

[57] Q. L. Fan and F. M. Liu, "Studying of relationship between ambulatory blood pressure of hypertension and syndrome differentiation type of TCM," Jilin Journal of TCM, vol. 30, no. 10, pp. 859-860, 2010.

[58] W. Zhu, S. J. Li, and X. M. Ruan, "Study on the relationship between arterial compliance and syndrome differentiation typing in hypertensive patients," Lishizhen Medicine and Materia Medica Research, vol. 20, no. 1, pp. 38-40, 2009.

[59] F. M. Liu, X. H. Chen, W. Q. Du et al., "Clinical research on the distribution regularity of TCM syndrome types," Journal of Chinese Medicine in Jiangsu, vol. 41, no. 10, pp. 33-34, 2009.

[60] S. Y. He, D. Y. Fu, and L. H. Zu, "Correlation of traditional Chinese medicine syndromes with B-type natriuretic peptide and the risk of stroke in patients with essential hypertension: a preliminary study," China Journal of Traditional Chinese Medicine and Pharmacy, vol. 28, no. 1, pp. 281-284, 2013.

[61] J. Y. Tang, Y. H. Wang, N. Tang et al., "Correlativity between Patterns of Hypertension in TCM and Polymorphism of COX2," Shanghai Zhongyiyao Daxue Xuebao, vol. 26, no. 3, pp. 32-35, 2012.

[62] N. J. Gong, Q. Li, J. Chen et al., "A correlated study between TCM syndrome and cardiac structural functional changes in patients with primary hypertension," Tianjin Journal of Traditional Chinese Medicine, vol. 27, no. 1, pp. 19-21, 2010.

[63] C. Zhang, Z. H. Xing, W. P. Liu et al., "Relativity investigation of plasma endothelin and blood pressure in patients with hypertension with different traditional Chinese medical classification," Liaoning Journal of TCM, vol. 32, no. 1, pp. 6-8, 2005.

[64] F. M. Liu, W. Q. Du, L. Shen et al., "A correlated study between left ventricular hypertrophy of hypertension and TCM syndrome type," Liaoning Journal of TCM, vol. 44, no. 12, pp. 871-872, 2009.

[65] J. Y. Wang, "A correlated study between syndrome differentiation type of TCM and ambulatory blood pressure," Medical Laboratory Sciences, vol. 3, pp. 143-144, 2012.

[66] M. M. Yao and T. J. Huang, "A correlated study between syndrome differentiation type of TCM and blood uric acid," Fujian Journal of TCM, vol. 38, no. 5, pp. 9-11, 2007.

[67] L. L. Guo, Y. Zhou, and T. T. Zhuang, "Relationship between the endothelin/nitric oxide and hypertension with traditional Chinese medical classification," Guizhou Medical Journal, vol. 26, no. 6, pp. 502-504, 2002.

[68] J. Z. Zhang, L. G. Chen, X. Q. Hu et al., "Influence of astragalus polysaccharide on the expression of Toll-like receptor 4 and nuclear transcription factor- $\mathrm{k}$ B in essential hypertension patients with blood stasis syndrome," Journal of Traditional Chinese Medicine, vol. 52, no. 15, pp. 1286-1304, 2011.

[69] W. Q. Liao, W. M. Huang, B. He et al., "Observation on insulin resistance of essential hypertension treated by the therapeutic method of activating blood circulation to dissipate Blood Stasis," Chinese Journal of Information on TCM, vol. 17, no. 9, pp. 9-11, 2010.

[70] Y. W. Xiong, "Clinical effect of the modified banxia baizhu tianma decoction combining western medicine on 60 patients with phlegm-dampness type primary hypertension," Chinese Medicine Modern Distance Education of China, vol. 8, no. 13, pp. $67-68,2010$.

[71] C. X. Jiang, J. M. Cao, and J. Y. Xu, "Clinical research on effects of the renin-angiotensin-II of senile patients with high blood pressure by jianling decoction," Chinese Journal of Science and Technology of Chinese Medicine, vol. 19, no. 2, 2012.

[72] Z. C. Huang and Q. P. Wei, "Curative effect analysis on identifying treatment of resistant hypertension combined with the system of traditional Chinese medicine," Chinese Community Doctors, vol. 14, no. 35, pp. 192-193, 2012.

[73] X. Lu, "Clinical research on the treatment of patients with liverkidney yin deficiency of hypertension by qiju dihuang wan decoction," Xinjiang Journal of Chinese Medicine, vol. 22, no. 5, pp. 20-22, 2004.

[74] H. T. Sun and C. Wang, "Different manifestations of cognitive dysfunction in hypertensive patients with different syndromes of traditional Chinese medicine," Chinese Journal of Clinical Rehabilitation, vol. 9, no. 20, pp. 63-65, 2005.

[75] Y. Xiang, G. C. Bai, and L. M. Wu, "Syndrome differentiation type and of nursing modern of community of primary hypertension," Hebei Journal of TCM, vol. 34, no. 5, pp. 766-768, 2012.

[76] D. X. Zhu, "The effect of injected Ligustrazine to shenshu point in patients with hypertension," China Practical Medicine, vol. 4, no. 15 , pp. 38-40, 2009.

[77] C. Y. Xu and F. L. Wang, "Clinical observation of hypertensive effect of taichong acupoint injection with ligustrazine on hypertension," Occupations and Health, vol. 25, no. 18, pp. 20002002, 2009.

[78] M. Lin and N. S. Kang, "Study on carotid ultrasound of phlegm dampness syndromes compared with yang hyperactivity syndromes of not intervention in primary hypertension," Journal of Shandong University of TCM, vol. 36, no. 2, pp. 115-117, 2012. 
[79] H. Feng, Z. C. Liu, and B. Xu, "Clinical observation on the treatment of 36 cases of excessive accumulation of phlegmdampness of essential hypertension complicated by obesity by warm acupuncture," Journal of Anhui TCM College, vol. 32, no. 1, pp. 47-50, 2013.

[80] A. W. Yu and E. H. Xing, "Clinical observation on the treatment of 90 cases with hypertension by Xifeng Tongluo Huayu decoction," Journal of Sichuan of TCM, vol. 28, no. 3, pp. 74-76, 2010.

[81] C. Qiu, X. D. Cheng, F. K. Cheng et al., "Clinical observation on the treatment of senile hypertension by Yiqihuoxue decoction," Hebei Journal of TCM, vol. 33, no. 6, pp. 838-839, 2011.

[82] T. C. Wu and T. Xu, "Clinical observation on the treatment of 60 cases of senile hypertension by tonifying qi and yin," Yunnan Journal of TCM, vol. 33, no. 4, pp. 31-33, 2012.

[83] X. M. Fang, X. Y. Huang, Q. Wang et al., "Research of clustering analysis of syndrome differentiation type of hypertension," Guangxi Journal of TCM, vol. 30, no. 5, pp. 9-11, 2007.

[84] W. Fang, T. L. Chen, and G. L. Zhu, "Research on the syndrome differentiation type of primary hypertension and the characteristics of dynamic blood pressure changes," Zhejiang Journal of TCM, vol. 4, pp. 164-166, 2003.

[85] L. L. Peng and D. Z. Shi, "Analysis on syndromes elements of primary hypertension with depression," Beijing Journal of TCM, vol. 29, no. 7, pp. 534-536, 2010.

[86] H. Y. Yang, Y. R. Jin, and H. Yang, "Relationship between the syndrome differentiation type of hypertension and ambulatory blood pressure," Chinese Journal of Information TCM, vol. 11, no. 1, pp. 23-46, 2004.

[87] X. L. Shi, Z. L. Wen, N. Zhang et al., "Clinical investigation on related factors and rule of the type of distribution of primary hypertension with depression," Jiangsu Journal of TCM, vol. 45, no. 2, pp. 25-26, 2013.

[88] Y. M. Han, "Clinical study on the treatment of primary hypertension based on syndrome differentiation of TCM," Health Guides of the International Medical, vol. 10, no. 10, pp. 120-122, 2004.

[89] R. Shen, Y. D. Chen, Z. X. Zhang et al., "Exploring the correlation of syndrome differentiation of primary hypertension and heart rate variability," China Journal of Traditional Chinese Medicine and Pharmacy, vol. 23, no. 5, pp. 453-455, 2008.

[90] Y. Shen, J. D. Zhang, L. H. Hu et al., “The relatirity of insulin resistance and syndrome differentiation typing in essential hypertension," Journal of Shandong University, vol. 43, no. 2, pp. 142-145, 2005.

[91] X. L. Liu, A. L. Wei, F. Luo et al., "Ultrasound study of carotid artery plaque score and the carotid intinamedia thickness in essential hypertension with traditional chinese medical classification," Liaoning Journal of TCM, vol. 36, no. 5, pp. 689-692, 2009.

[92] X. Lu, C. Y. Wei, L. H. Yang et al., "Study on the relationship between syndrome type of TCM of primary hypertension and apolipoprotein," Journal of Guangxi Traditional Chinese Medical University, vol. 14, no. 3, pp. 4-6, 2011.

[93] K. F. Guo, J. F. Zhang, and W. Q. Yang, "Relationships between personality characteristic and diff erentiation of symptom and sign for classification of syndrome of traditional Chinese medicine in patients with primary hypertension," Chinese Journal of Cardiovascular Rehabilitation Medicine, vol. 15, no. 4, pp. 326-328, 2006.

[94] J. P. Zhang, H. W. Yuan, H. L. Wang et al., "Correlation between concentration of $\beta 2$ microglobulin in blood and urine in patients with primary hypertension and traditional Chinese medicine syndrome," Hebei Journal of TCM, vol. 34, no. 5, pp. 658-660, 2012.

[95] C. W. Dong, M. Dong, Q. S. Xing et al., "Relationship between polymorphism of angiotensin converting enzyme gene and different traditional Chinese medicine syndrome in patients with essential hypertension," Chinese Journal of Pathophysiology, vol. 26, no. 8, pp. 1545-1548, 2010.

[96] Z. B. Zhang, C. G. Zhou, and S. Lu, "Distribution of TCM syndrome types of essential hypertension and their relationship to biochemical indicators," Liaoning Journal of TCM, vol. 37, no. 6, pp. 969-972, 2010.

[97] J. Chen and Z. Q. Chen, "Clinical study on characteristics of changes in dynamic blood pressure in patients with primary hypertension of blood stasis syndrome," World Journal of Integrated Traditional and Western Medicine, vol. 3, no. 10, pp. 600-603, 2008.

[98] L. Liu and Y. Q. Zhang, "A correlated study between HCY, MAU and TCM syndrome type of hypertension," Heilongjiang Journal of TCM, vol. 5, pp. 5-7, 2010.

[99] X. Zhou, "Clinical effect analysis on treating 86 cases of essential hypertension with treatment based on differentiation of symptoms and signs," Clinical Journal of Chinese Medicine, vol. 5, no. 3, pp. 55-57, 2013.

[100] G. Y. Chen, L. J. Wang, J. Liu et al., "Relationship between TCM syndrome types and related risk factors of essential hypertension," Chinese Journal of Information on TCM, vol. 19, no. 11, pp. 16-18, 2012.

[101] W. Q. Zhou, D. H. Liu, Y. N. Dai et al., "Study on the correlation between the related factors and TCM syndromes of essential hypertension with left ventricular hypertrophy," Journal of Traditional Chinese Medicine, vol. 50, no. 3, pp. 248-250, 2009.

[102] Y. X. Ding, Y. X. Zhou, B. Liu et al., "Preliminary analysis on epidemiological characteristics of TCM syndromes of primary hypertension in Guangdong region," Journal of Anhui TCM College, vol. 28, no. 2, pp. 25-27, 2009.

[103] Z. W. Li, "Clinical observation on the treatment of 125 cases of essential hypertension complicated by syndrome differentiation and treatment of TCM," Forum on Traditional Chinese Medicine, vol. 17, no. 2, pp. 31-33, 2002.

[104] Z. F. Qiu, "Clinical observation on the treatment of 97 cases of essential hypertension by syndrome differentiation of TCM," Chinese Journal of Ethnomedicine and Ethnopharmacy, vol. 3, pp. 187-189, 2010.

[105] W. L. Deng and G. Zhao, "Clinical observation on syndrome differentiation of TCM of 80 cases of the hypertension patients with left ventricular hypertrophy," Xinjiang Journal of TCM, vol. 22, no. 5, pp. 20-22, 2004.

[106] Q. Chen, "Clinical study on primary hypertension early time kidney harm with Zishuiqingganyin," Chinese Archives of TCM, vol. 28, no. 6, pp. 1332-1335, 2010.

[107] T. W. Zhang and B. R. Zheng, "Clinical observation on the treatment of 30 cases of essential hypertension with liver-yang hyperactivity by “Jiangya 2'”' Fujian Medical Journal, vol. 31, no. 6, pp. 133-135, 2009.

[108] Y. X. Wan and T. Z. Zhang, "Research on relationship between angiotensinogen gene M235T and TCM syndrome type in essential hypertension patients," Chinese Journal of Integrated Traditional and Western Medicine, vol. 28, no. 1, pp. 36-38, 2008.

[109] Y. J. Xu and T. Z. Zhang, "Relationship of day and night rhythm of Essential Hypertension with TCM Syndrome patterns," Journal of New Chinese Medicine, vol. 40, no. 5, pp. 41-43, 2008. 
[110] D. Zhang and H. R. Li, "Epidemiological investigation and distribution of TCM syndrome of primary hypertension in community," The Community of Chinese Medicine, vol. 12, no. 229, p. 83, 2010.

[111] Z. H. Pu, G. D. Yang, T. Y. Ding et al., "Clinical observation on the effect of blood pressure, cholesterol, blood sugar, and the clinical syndromes of traditional Chinese medicine of 220 cases of essential hypertension by "Ping ganyin" capsule," China Journal of Chinese Materia Medica, vol. 33, no. 10, pp.1202-1205, 2008.

[112] W. J. Cheng, Z. C. Tan, F. Guo et al., "Epidemiological studies on TCM syndrome of 602 cases of primary hypertension," Chinese Journal of Integrated Traditional and Western Medicine, vol. 13, no. 4, pp. 261-263, 2003.

[113] B. Y. Ding, Z. B. Shao, L. Zheng et al., "Study on the relationship between early nephritic injures by essential hypertension and traditional Chinese medicine syndrome patterns," Journal of Anhui TCM College, vol. 5, no. 4, pp. 6-8, 2006.

[114] S. Fu and Y. L. Li, "Establishing standards of measuring essential hypertension with hyperactive of liver-Yang syndrome based on multivariate analysis of statistics," Journal of Shandong University of TCM, vol. 34, no. 1, pp. 14-16, 2010.

[115] Q. Pan, Z. D. Liu, L. P. Chen et al., "Shiliangcha Prescription of "She medicine" combined with western medicine in treating essential hypertension of damp-phlegm pattern: a report of 30 cases," Shanghai Journal of TCM, vol. 46, no. 3, pp. 49-51, 2012.

[116] G. L. Chen, B. Y. Wang, H. P. Liu et al., "Survey on traditional Chinese medicine syndrome types in 471 patients with essential hypertension," Journal of Anhui TCM College, vol. 30, no. 4, pp. 24-26, 2011.

[117] Y. H. Zhao, Y. D. Liu, P. G. Huang et al., "Effects of clinical treatment and protection on endothelial function of early essential hypertension's patients treated with YNJY soup in southern Guangdong area," China Journal of Traditional Chinese Medicine and Pharmacy, vol. 24, no. 9, pp. 1148-1150, 2009.

[118] C. G. Zhou, Z. B. Zhang, C. X. Xia et al., "Association of the C344T polymorphism of CY P11 B2 gene with TCM syndrome type in essential hypertension," Liaoning Journal of TCM, vol. 37, no. 4, pp. 577-579, 2010.

[119] J. H. Huang, Q. S. Zheng, R. Gao et al., "Clinical equivalence evaluation on the efficacy and safety of Niuhuang jiangya tablets and pills in the treatment of patients with primary hypertension (overabundant liver-fire)," Chinese Journal of Evidence-Based Medicine, vol. 4, no. 4, pp. 249-251, 2004.

[120] X. Lu, "Clinical observation on the treatment of essential hypertension by compound Danshen dripping pills," Journal of Guangxi Traditional Chinese Medical University, no. 2, pp. 2829, 2004.

[121] D. Zhu and S. B. Lin, "Clinical observation on the treatment of essential hypertension with yin deficiency and yang excess by Zishui pinggan decoction," Chinese Journal of Geriatric Care, vol. 10, no. 4, pp. 32-35, 2012.

[122] C. H. Wang, Y. Gao, J. Z. Chen et al., "Impact analysis of syndrome differentiation combined with modified Zixie decoction for hypertension symptoms," World Chinese Medicine, vol. 8, no. 3, pp. 285-287, 2013.

[123] D. G. Ji, "Ling kok uncaria decoction in the treatment of hypertension," Journal of Chinese Herbs of TCM, vol. 18, no. 10, pp. 92-93, 2011.

[124] L. Yuan, D. Tu, X. Ye, and J. Wu, "Hypoglycemic and hypocholesterolemic effects of Coptis chinensis franch inflorescence,"
Plant Foods for Human Nutrition, vol. 61, no. 3, pp. 139-144, 2006.

[125] H. J. Koo, K. H. Lim, H. J. Jung, and E. H. Park, "Antiinflammatory evaluation of gardenia extract, geniposide and genipin," Journal of Ethnopharmacology, vol. 103, no. 3, pp. 496500, 2006.

[126] S. C. Ho, Y. F. Ho, T. H. Lai, T. H. Liu, and R. Y. Wu, "Traditional Chinese herbs against hypertension enhance the effect of memory acquisition," American Journal of Chinese Medicine, vol. 33, no. 5, pp. 787-795, 2005.

[127] J. Zhou and S. Zhou, "Antihypertensive and neuroprotective activities of rhynchophylline: the role of rhynchophylline in neurotransmission and ion channel activity," Journal of Ethnopharmacology, vol. 132, no. 1, pp. 15-27, 2010.

[128] K. Hansen, U. Nyman, U. W. Smitt et al., "In vitro screening of traditional medicines for anti-hypertensive effect based on inhibition of the angiotensin converting enzyme (ACE)," Journal of Ethnopharmacology, vol. 48, no. 1, pp. 43-51, 1995.

[129] J. Gu, J. J. Wang, J. Yan et al., "Effects of lignans extracted from Eucommia ulmoides and aldose reductase inhibitor epalrestat on hypertensive vascular remodeling," Journal of Ethnopharmacology, vol. 133, no. 1, pp. 6-13, 2011.

[130] L. Li, J. Yan, K. Hu et al., "Protective effects of Eucommia lignans against hypertensive renal injury by inhibiting expression of aldose reductase," Journal of Ethnopharmacology, vol. 139, no. 2, pp. 454-461, 2012.

[131] F. Greenway, Z. Liu, Y. Yu, and A. Gupta, "A clinical trial testing the safety and efficacy of a standardized Eucommia ulmoides oliver bark extract to treat hypertension," Alternative Medicine Review, vol. 16, no. 4, pp. 338-347, 2011.

[132] Y. P. Han, N. S. Wang, S. Q. Mi, and Q. D. Liu, "Effect of Wuling Powder on rats with renal hypertension," Zhong Xi Yi Jie He Xue Bao, vol. 1, no. 4, pp. 285-288, 2003.

[133] J. Y. Chen, H. L. Fan, and S. F. Zhang, "Effect of modified Zexie decoction on prevention of kidney injuries of rats with hypertension induced by high-salt diet," Zhong Yi Za Zhi, vol. 53, no. 3, pp. 234-237, 2012.

[134] S. J. Wu, L. T. Ng, and C. C. Lin, "Antioxidant activities of some common ingredients of traditional Chinese medicine, Angelica sinensis, Lycium barbarum and Poria cocos," Phytotherapy Research, vol. 18, no. 12, pp. 1008-1012, 2004.

[135] Y. Sun, S. Wang, T. Li, X. Li, L. Jiao, and L. Zhang, "Purification, structure and immunobiological activity of a new water-soluble polysaccharide from the mycelium of Polyporus albicans (Imaz.) Teng," Bioresource Technology, vol. 99, no. 4, pp. 900-904, 2008.

[136] R. Yuan and Y. Lin, "Traditional Chinese medicinean approach to scientific proof and clinical validation," Pharmacology and Therapeutics, vol. 86, no. 2, pp. 191-198, 2000.

[137] W. Y. Jiang, "Therapeutic wisdom in traditional Chinese medicine: a perspective from modern science," Trends in Pharmacological Sciences, vol. 26, no. 11, pp. 558-563, 2005.

[138] D. D. Kim, F. A. Sánchez, R. G. Durán, T. Kanetaka, and W. N. Durán, "Endothelial nitric oxide synthase is a molecular vascular target for the Chinese herb Danshen in hypertension," American Journal of Physiology-Heart and Circulatory Physiology, vol. 292, no. 5, pp. H2131-H2137, 2007.

[139] Y. Z. Hou, G. R. Zhao, Y. J. Yuan, G. G. Zhu, and R. Hiltunen, "Inhibition of rat vascular smooth muscle cell proliferation by extract of Ligusticum chuanxiong and Angelica sinensis," Journal of Ethnopharmacology, vol. 100, no. 1-2, pp. 140-144, 2005. 
[140] R. L. Simeonova, V. B. Vitcheva, M. S. Kondeva-Burdina, I. N. Krasteva, S. D. Nikolov, and M. K. Mitcheva, "Effect of purified saponin mixture from Astragalus corniculatus on enzyme- and non-enzyme-induced lipid peroxidation in liver microsomes from spontaneously hypertensive rats and normotensive rats," Phytomedicine, vol. 17, no. 5, pp. 346-349, 2010.

[141] B. Xue, J. Li, Q. Chai, Z. Liu, and L. Chen, "Effect of total flavonoid fraction of Astragalus complanatus R. Brown on angiotensin II-induced portal-vein contraction in hypertensive rats," Phytomedicine, vol. 15, no. 9, pp. 759-762, 2008.

[142] L. M. Yao, T. W. Liu, W. F. Wu et al., "Effects of Astragalus injection in reversing left ventricular hypertrophy induced by renal hypertension in rats," Zhongguo Zhong Xi Yi Jie He Za Zhi, vol. 29, no. 10, pp. 918-921, 2009.

[143] L. Z. Lin, X. G. He, L. Z. Lian, W. King, and J. Elliott, "Liquid chromatographic-electrospray mass spectrometric study of the phthalides of Angelica sinensis and chemical changes of Zligustilide," Journal of Chromatography A, vol. 810, no. 1-2, pp. 71-79, 1998

[144] Y. Z. Hou, G. R. Zhao, J. Yang, Y. J. Yuan, G. G. Zhu, and R. Hiltunen, "Protective effect of Ligusticum chuanxiong and Angelica sinensis on endothelial cell damage induced by hydrogen peroxide," Life Sciences, vol. 75, no. 14, pp. 1775-1786, 2004. 


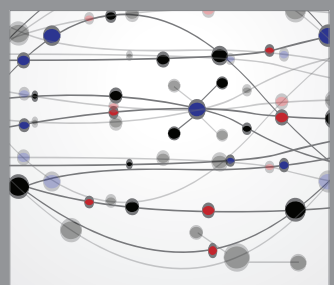

The Scientific World Journal
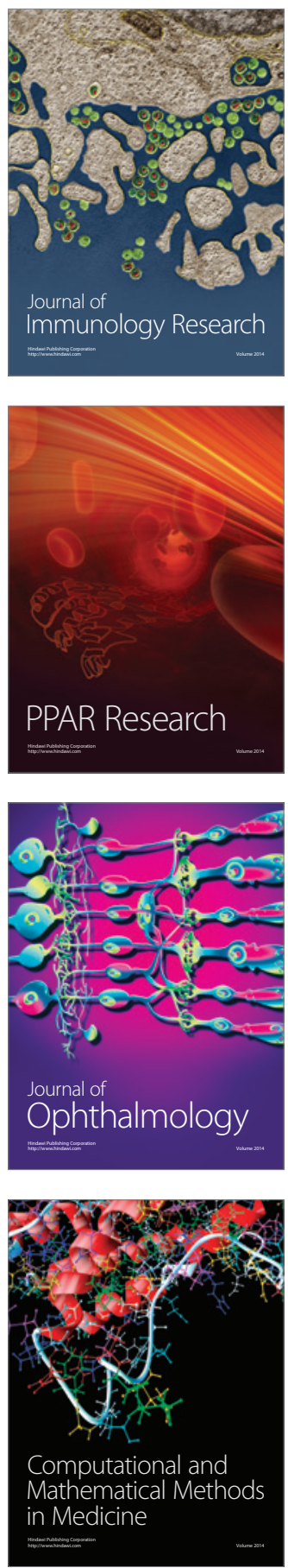

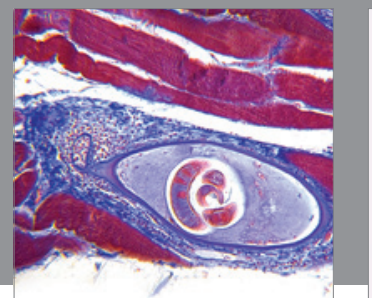

Gastroenterology

Research and Practice
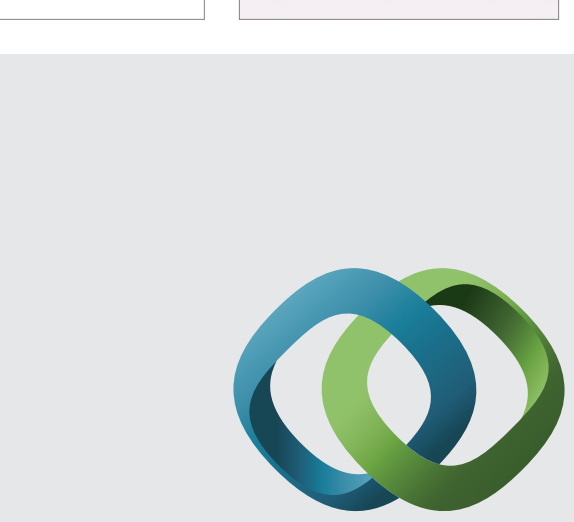

\section{Hindawi}

Submit your manuscripts at

http://www.hindawi.com
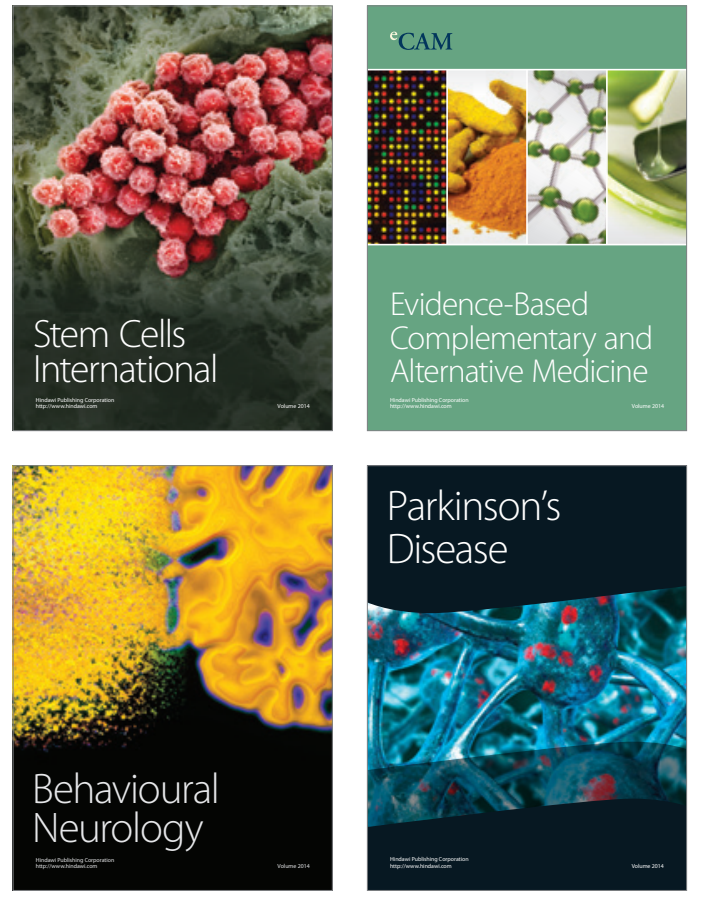
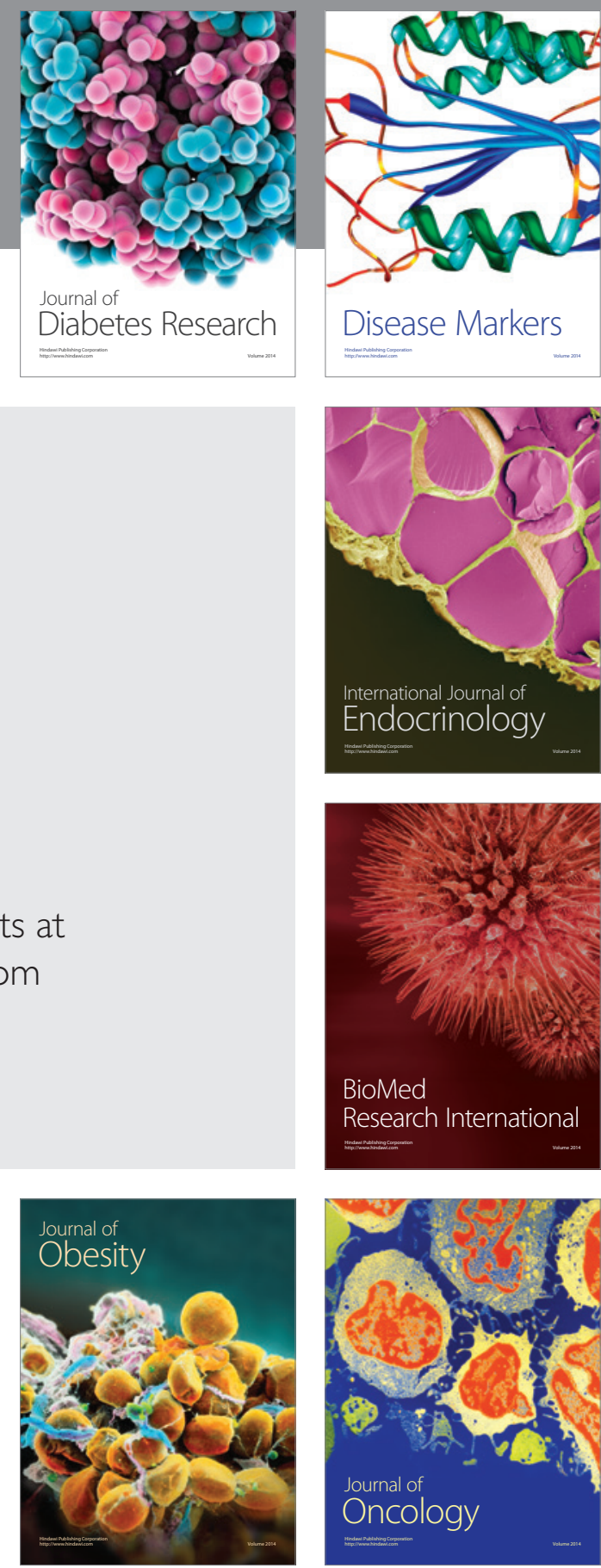

Disease Markers
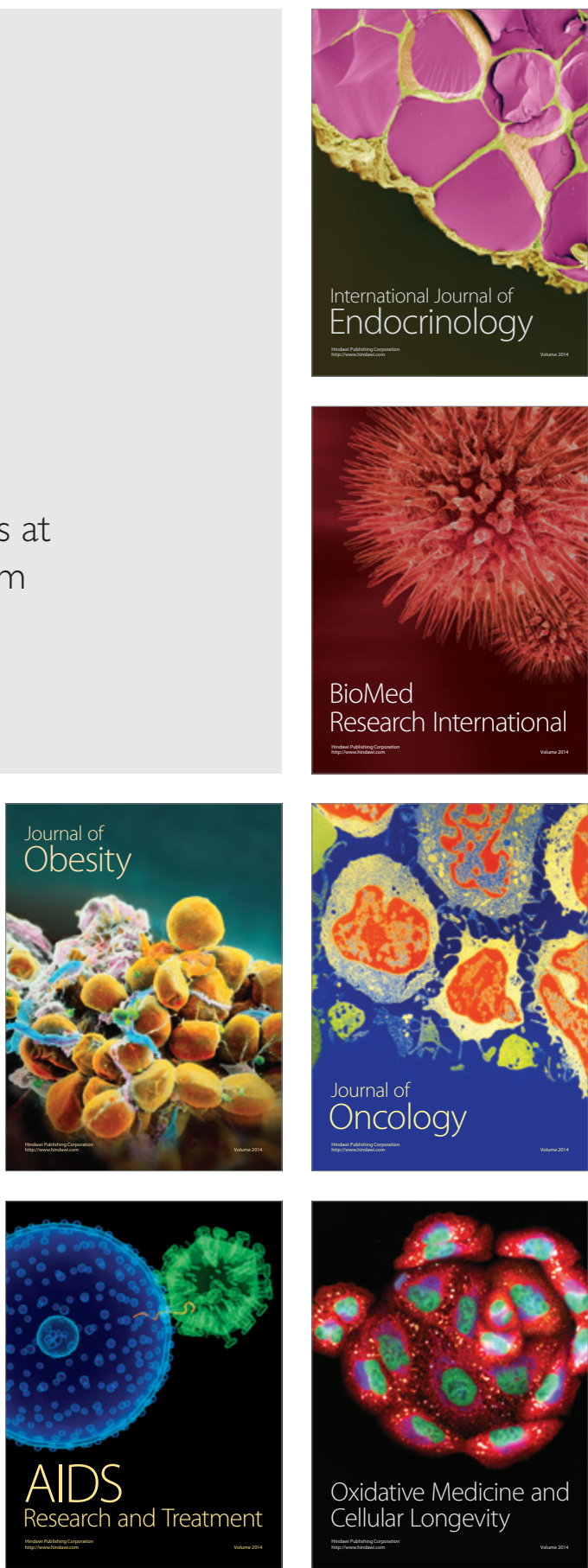